\title{
Gromov-Witten invariants and rigidity of Hamiltonian loops with compact support on noncompact symplectic manifolds
}

\author{
GuAngCun LU ${ }^{1}$
}

\begin{abstract}
In this paper the Gromov-Witten invariants on a class of noncompact symplectic manifolds are defined by combining Ruan-Tian's method with that of McDuff-Salamon. The main point of the arguments is to introduce a method dealing with the transversality problems in the case of noncompact manifolds. Moreover, the techniques are also used to study the topological rigidity of Hamiltonian loops with compact support on a class of noncompact symplectic manifolds.
\end{abstract}

\section{Introduction.}

Since Gromov introduced his celebrated pseudo-holomorphic theory on symplectic manifolds in 80's ([Gr]), many important questions in symplectic geometry and related fields have been solved. In particular, Witten [W1, W2] pointed out that Gromov's study of the moduli space of holomorphic curves could be used in principle to describe correlation functions in the topological quantum field theory. The moduli spaces of holomorphic spheres were used by Ruan to define certain symplectic invariants of semi-positive symplectic manifolds $([R 1])$. In the semi-positive closed symplectic manifolds the more general Gromov-Witten invariants of any genus, including so called mixed invariants, were constructed in [RT1] and later [RT2] and thus they gave the first rigorous mathematics theory of quantum cohomology. This forms a solid mathematical basis for the topological sigma model. In addition, they also applied these invariants to the Mirror Symmetry Conjecture, the Enumerative Geometry and Symplectic topology. It should be noted that in this case their mixed invariants are of integral values.

On the other hand the Gromov-Witten invariants were studied axiomatically in $[\mathrm{KM}]$. More recently, the Gromov-Witten invariants for any projec-

\footnotetext{
${ }^{1}$ Supported by the NNSF 19971045 and ETPME of China.
} 
tive manifolds (cf. [LT1]) and any closed symplectic manifolds were defined (cf. [FO], [LT2], [R3], [Sie]).

It has been expected that the Gromov-Witten invariants should also be defined for noncompact symplectic manifolds and families of symplectic manifolds(cf. [K1, p. 364]). In fact, the latter was carried out in [L], [R3]. Roughly saying, if $p: Y \rightarrow M$ is an oriented fiber bundle such that the fiber $X$ and the base $M$ are smooth, compact, oriented manifolds (which implies that $Y$ is also such a manifold), and $\omega$ is a closed 2-form on $Y$ such that $\omega$ restricts to a symplectic form over each fiber, then $Y$ can be viewed as a family of symplectic manifolds and the Gromov-Witten invariants over $Y$ are defined in [R3]. However, for noncompact symplectic manifolds $(V, \omega)$ how the Gromov-invariants over them should be defined, we so far do not see it in the literatures. Generally speaking, the key points in many applications of the Gromov's pseudo-holomorphic curve theory are the compactness problems. On the closed symplectic manifolds one have obtained very good results (cf. [Gr], [RT1], [PW], [Ye]). For the general noncompact symplectic manifolds (even without boundary) these problems become very complicated. In this paper we define the Gromov-Witten invariants on a class of special noncompact symplectic manifolds-semi-positive geometrically bounded one. Precisely speaking, we generalize the main results in [RT1] to this class of symplectic manifolds. The notion of geometrically bounded (abb. g. bounded) symplectic manifolds was first appeared in [Gr]. This kind of manifolds has many nice properties so that many results on closed symplectic manifolds can be extended on them in some reasonable ways (see $\S 2$ ).

However, since $V$ is noncompact, for every integer $m \geq 1$ the Banach manifolds $\mathcal{J}_{\tau}^{m}$ consisting of all $C^{m}$-smooth $\omega$-tame almost complex structures on $(V, \omega)$ and the group $\operatorname{Diff}^{m}(V)$ of all $C^{m}$-diffeomorphisms on $V$ are not separable, and thus neither are some correspondent moduli spaces separable. Hence it is difficult using Sard-Smale theorem in many transversality arguments. One may wish to use its generalization version due to Quinn to replace it. But this requires the Fredholm map considered to be proper or $\sigma$-proper. Under our case it can not be satisfied. On the other hand, for a given $J \in \mathcal{J}_{\tau}^{m}(M, \omega)$ the space $C^{m}\left(\mathrm{~T}_{J}\right)$ of all $C^{m}$-sections does not gives rise to a local model for the space $\mathcal{J}_{\tau}^{m}(V, \omega)$ via $Y \mapsto J \exp (-J Y)$. To see this point, note that $J \in \mathcal{J}_{\tau}^{m}(V, \omega)$ only means $\omega(\xi, J(p) \xi)>0$ for every $p \in V$ and $\xi \in T_{p} V \backslash\{0\}$ and from $\|Y\|_{C^{m}}<\delta$ it does not follow that $\|Y\|_{C^{0}}<\eta$ which is an arbitrary given positive number smaller than $\delta$. Thus even if for every $p \in V$ and $\xi \in T_{p} V \backslash\{0\}$ we can obtain $\omega(\xi, J(p) \exp (-J(p) Y(p)) \xi)>0$ as $|Y(p)|$ sufficiently small, but due to the 
noncompactness of $V$ one can not derive that for a given smooth nowhere null vector field $\zeta$ on $V, \omega(\zeta(p), J(p) \exp (-J(p) Y(p)) \zeta(p))$ is more than zero at all points $p \in V$ whether $\|Y\|_{C^{m}}$ is small. In order to overcome these difficulties we construct suitable separable Banach manifolds to replace the Banach manifolds chosen naturally in the case of compact manifolds. In $\S 2$ and $\S 4$ these techniques are all used. The method may probably applied to generalize other results on compact manifolds in symplectic geometry and Seiberg-Witten invariants theory to noncompact manifolds.

In our case replacing $H^{*}(V, \mathbb{Z})$ by $H_{*}(V, \mathbb{Z})$ the homology we can show that there is an quantum ring structure on it. In contrast to the case of closed symplectic manifolds it seem to be very hard to use the recent techniques developed by [FO], [LT1, LT2], [R3], [Sie] to define the Gromov-Witten invariants on all noncompact compact g. bounded symplectic manifolds because of the technical difficulties.

Inspired by Seidel's work [Se1] the quantum homology is also used to study topological rigidity of Hamiltonian loops by F.Lalonde, D. McDuff and L. Polterovich in [LMP]. Precisely speaking, they proved that if $\omega_{1}$ and $\omega_{2}$ are two symplectic forms satisfying certain monotonicity assumptions on a closed manifold $M$ then every loop $\phi=\left\{\phi_{t}\right\}_{0 \leq t \leq 1}$ in the group $\operatorname{Ham}\left(M, \omega_{1}\right) \cap \operatorname{Symp}\left(M, \omega_{2}\right)$ can be homotoped in $\operatorname{Symp}\left(M, \omega_{2}\right)$ to a loop in $\operatorname{Ham}\left(M, \omega_{2}\right)$. Combing their ideas with our techniques together we generalize their results to the case of the Hamiltonian loops with compact support on a class of noncompact g.bounded symplectic manifolds in Corollary 6.2. Moreover, as a consequence the corresponding result on compact symplectic manifolds with contact type boundary is also obtained in Corollary 6.3. The main points of the arguments are to construct a kind of suitable closed twoforms on the Hamiltonian fibre bundle over $S^{2}$ with noncompact g.bounded symplectic manifolds as a fibre to replace the unique coupling class whose top power vanishes so that the composition rule may be obtained.

The arrangements of this paper are as follows. In $\S 2$ we give some basic definitions and lemmas in geometrically bounded symplectic manifolds, and specially a new technique on transversality arguments. In $\S 3$ we generalized the results of transversality and compactness to our case. Since the arguments are similar we only give the necessary improvements. The Gromov-Witten invariants are defined in $\S 4$. As a consequence we also define the Gromov-Witten invariants of compact symplectic manifolds with contact type boundary in $\S 5$. In $\S 6$ the study of the topological rigidity of Hamiltonian loops with compact support on noncompact g.bounded symplectic manifolds with the weaker semi-positivity assumptions is given. In final Appendix a theorem which characterizes the Hamiltonian symplecto- 
morphisms on a compact symplectic manifold with contact type boundary in terms of the flux homomorphism is provided.

Acknowledgements. This revised version including the topological rigidity of Hamiltonian loops was accomplished during the author's visit at IHES. The author would like to express his thanks to Professor J.Bourguignon for his invitation and hospitality. He thanks very much Dr. Paul Seidel for carefully checking the original version, correcting many mistakes and stimulating conversations. He is also grateful to Professors Gang Tian and Yongbin Ruan for their kind explanations to me with respect to their papers. Finally, I wish to thank the referee for telling me another possible simpler method to achieve the transversality in the definition of our Gromov-Witten invariants.

\section{Definitions and Lemmas.}

In this section we give some necessary technical lemmas. Notice first that the following conclusions in Riemannian geometry are some easy exercises.

Lemma 2.1. Let $(M, g)$ be a Riemannian manifold with injectivity radius $i(M, g)>0$. Then it is complete and for any compact subsets $K$ in $M$ and arbitrary $\varepsilon>0$,

$$
K_{\varepsilon}=\left\{p \in M: d_{g}(p, K) \leq \varepsilon\right\}
$$

is compact. Here $d_{g}$ denote the distance induced by $g$.

Lemma 2.2. For the product Riemannian manifold $(M, g)=\left(M_{1}, g_{1}\right) \times$ $\left(M_{2}, g_{2}\right)$ we have

(i) $i(M, g)=\min \left\{i\left(M_{1}, g_{1}\right), i\left(M_{2}, g_{2}\right)\right\}$;

(ii) $\forall\left(m_{1}, m_{2}\right) \in M, u=\left(u_{1}, u_{2}\right)$, and $v=\left(v_{1}, v_{2}\right) \in T_{m} M$ it holds that

$$
K_{g}\left(\Pi_{m}\right)=\frac{1}{4}\left(K_{g_{1}}\left(\Pi_{m_{1}}\right)+K_{g_{2}}\left(\Pi_{m_{2}}\right)\right),
$$

where $\Pi_{m}=\operatorname{span}\{u, v\}, \Pi_{m_{1}}=\operatorname{span}\left\{u_{1}, v_{1}\right\}$ and $\Pi_{m_{2}}=$ $\operatorname{span}\left\{u_{2}, v_{2}\right\}$.

Next let us recall the following definition (cf. [ALP], [Gr], [Sik]).

Definition 2.3. Let $(V, \omega)$ be a symplectic manifold without boundary. Call it geometrically bounded if there exists an almost complex structure $J$ and a complete Riemannian metric $g$ on $V$ such that the following properties are satisfied: 
$1^{\circ} J$ is uniformly tamed by $\omega$, that is, there exist strictly positive constants $\alpha_{0}$ and $\beta_{0}$ such that

$$
\omega(X, J X) \geq \alpha_{0}\|X\|_{g}^{2} \quad \text { and } \quad|\omega(X, Y)| \leq \beta_{0}\|X\|_{g}\|Y\|_{g}
$$

for all $X, Y \in T V$.

$2^{\circ}$ The sectional curvature $K_{g} \leq C_{0}$ (a positive constant) and the injectivity radius $i(V, g)>0$.

Remark 2.4. By Lemma 2.1 we know that the requirement of the completeness for $g$ in Definition 2.3 is not necessary since this is actually contained in the condition $2^{\circ}$.

Clearly the closed symplectic manifolds are g. bounded, a product of two g. bounded symplectic manifolds is also such manifold. One can easily prove that every symplectic covering manifold of a $\mathrm{g}$. bounded symplectic manifold and every symplectic manifold without boundary which is isomorphic at infinity to the symplectization of a closed contact manifold are g. bounded. In [Lu2] we have proved that the cotangent bundles with respect to any twisted symplectic structures on it are g. bounded. In addition, one also should notice that any geometrically bounded symplectic manifolds are the tame almost complex manifolds in the sense of J.C. Sikorav (see [Sik]).

Given a closed Riemann surface $\Sigma$ with the complex structure $j$ and $J \in \mathcal{J}_{\tau}(V, \omega)$ we denote by $\overline{H o m}_{J}(T \Sigma, T V)$ the space of the smooth sections of the bundle of anti-J-linear homomorphisms from $T \Sigma$ to $T V$ over $\Sigma \times V$. Its element $\nu$ is called the inhomogeneous term. Recall that a smooth map $f: \Sigma \rightarrow V$ is called $(J, \nu)$-map if for any $z \in \Sigma$,

$$
\bar{\partial}_{J} f(z)=d f(z)+J(f(z)) \circ d f(z) \circ j(z)=\nu(z, f(z)) .
$$

In the following we only consider the inhomogeneous term $\nu$ satisfying

$$
\operatorname{Sup}_{(z, p) \in \Sigma \times V}\|\nu(z, p)\|_{\mathcal{L}\left(T_{z} \Sigma, T_{p} V\right)}<+\infty
$$

where the norm in $\mathcal{L}\left(T_{z} \Sigma, T_{p} V\right)$ is with respect to $g$ and the Riemannian metric on $\Sigma$ induced from $j$ and some area form. Notice that any two area forms on $\Sigma$ are proportional. The above condition is independent of the concrete choice of the compatible area forms.

Lemma 2.5. Let $(V, \omega, g, J)$ be as above Definition 2.3 , and $\sigma$ an area form on $\Sigma$ compatible with $j, \tau=\sigma \circ(i d \times j)$. Then for $N>0$ sufficiently large 
$(\Sigma \times V, \tilde{\omega}, \tau \oplus g, \tilde{J})$ is also a g.bounded symplectic manifold. Here $\tilde{\omega}=N \tau \times \omega$ and $\tilde{J}(z, p): T_{(z, p)}(\Sigma \times V) \rightarrow T_{(z, p)}(\Sigma \times V)$ is given by

$$
\left(X_{1}, X_{2}\right) \mapsto\left(j(z) X_{1}, J(p) \nu(z, p)\left(X_{1}\right)+J(p) X_{2}\right) .
$$

The proof of this lemma is an easy exercise. In fact, one can choose $\left(\alpha_{1}, \beta_{1}\right)$ to replace $\left(\alpha_{0}, \beta_{0}\right)$. Here $\alpha_{1}=\alpha_{0} / 2, \beta_{1}=2 \beta_{0}+\alpha_{0}+\Gamma^{4} \beta_{0}^{4} / \alpha_{0} \eta$ and

$$
\operatorname{Sup}_{(z, p) \in \Sigma \times V}\|\nu(z, p)\|_{\mathcal{L}\left(T_{z} \Sigma, T_{p} V\right)} \leq \Gamma<+\infty, \quad N \geq \frac{\alpha_{0}}{2}+\frac{\Gamma^{4} \beta_{0}^{4}}{2 \alpha_{0}^{2}} .
$$

Proposition 2.6. Under assumptions of Lemma 2.5, if $K \subset V$ is a compact subset and $u: \Sigma \rightarrow V$ a smooth $(J, \nu)$-map representing $A \in H_{2}(V, \mathbb{Z})$ and intersecting with $K$, then

$$
\operatorname{Im}(f) \subset K_{\rho_{0}}
$$

where $\rho_{0}=\rho_{0}\left(\alpha_{0}, \beta_{0}, C_{0}, i(V, g), j, J, \nu, A, \sigma\right)$.

Proof. Write $W=\Sigma \times V$ and $\bar{u}: \Sigma \rightarrow W, z \mapsto(z, u(z))$. Then $\bar{u}$ is $\tilde{J}_{\text {- }}$ holomorphic and its image can intersect with $\widehat{K}:=\Sigma \times K$ if and only if the image of $u$ is intersecting with $K$. Combing this with the taming property we can estimate its area with respect to the metric $\tau \oplus g$ as follows:

$$
\begin{aligned}
\operatorname{Area}_{\tau \oplus g}(\bar{u}(\Sigma)) & \leq \frac{1}{\alpha_{1}} \int_{\Sigma} \bar{u}^{*} \tilde{\omega} \\
& =\frac{1}{\alpha_{1}} \int_{\Sigma} u^{*} \omega+\frac{N}{\alpha_{1}} \int_{\Sigma} \sigma \\
& =\frac{1}{\alpha_{1}}\langle\omega, A\rangle+\frac{N}{\alpha_{1}} \int_{\Sigma} \sigma
\end{aligned}
$$

Now, by Lemma 2.5 we have

$$
\begin{aligned}
\tilde{\omega}\left(\left(X_{1}, X_{2}\right), \tilde{J}_{\lambda}\left(X_{1}, X_{2}\right)\right) & \geq \frac{\alpha_{0}}{2}\left\|\left(X_{1}, X_{2}\right)\right\|_{\tau_{0} \oplus g}^{2} \\
\left|\tilde{\omega}\left(\left(X_{1}, X_{2}\right),\left(Y_{1}, Y_{2}\right)\right)\right| & \leq \beta_{1}\left\|\left(X_{1}, X_{2}\right)\right\|_{\tau \oplus g}\left\|\left(Y_{1}, Y_{2}\right)\right\|_{\tau \oplus g}
\end{aligned}
$$

for every $(z, p) \in W$ and $X=\left(X_{1}, X_{2}\right), Y=\left(Y_{1}, Y_{2}\right) \in T_{(z, p)} W$. Here $\left\|\left(X_{1}, X_{2}\right)\right\|_{\tau \oplus g}^{2}=\left\|X_{1}\right\|_{\tau}^{2}+\left\|X_{2}\right\|_{g}^{2}$. Moreover, by lemma 2.2 the sectional curvature and injectivity radius of $(\Sigma \times V, \tau \oplus g)$ satisfy

$$
K_{\tau \oplus g} \leq \frac{1}{4}\left(1+C_{0}\right)
$$


and

$$
i(\Sigma \times V, \tau \oplus g)=\min \{i(\Sigma, \tau), i(V, g)\}
$$

respectively. Next, according to the comments below Definition 4.1.1 in [Sik], in our case we may take $C_{1}=1 / \pi, C_{2}=\beta_{1} / \alpha_{1}, \omega_{x} \equiv \tilde{\omega} / \beta_{1}$ and $r_{0}=\min \left(i(W, \tau \oplus g), 2 \pi / \sqrt{1+C_{0}}\right)$ such that the following monotonicity holds:

For a compact Riemannian surface $S$ with boundary and $\tilde{J}$ holomorphic map $f: S \rightarrow W$, if $f(S) \subset B(x, r) \subset W$, $f(\partial S) \subset \partial B$ and $x \in f(S)$ for some $r \leq r_{0}$, then

$$
\operatorname{Area}_{g}(f(S)) \geq \frac{\pi \alpha_{1}}{4 \beta_{1}} r^{2}
$$

From these and the proof of Proposition 4.41 in [Sik] it follows that

$$
\operatorname{Im}(\bar{u}) \subset U\left(\widehat{K}, C_{6} \operatorname{Area}(\operatorname{Im}(\bar{u}))\right),
$$

where $\widehat{K}=\Sigma \times K$ and $C_{6}=4 C_{1} C_{2} / r_{0}=4 \beta_{1} / \pi \alpha_{1} r_{0}$. Using the argument below Lemma 2.5 and an easy computation we can get

$$
C_{6}=\frac{4 \alpha_{0}^{3} \beta_{0}+2 \alpha_{0}^{4}+2 \beta_{0}^{4} \Gamma^{4}}{\pi \alpha_{0}^{4} \min \left(i(\Sigma, \tau), i(V, g), 2 \pi / \sqrt{1+C_{0}}\right)} .
$$

Notice that

$$
\operatorname{Area}(\bar{u}(\Sigma)) \leq \frac{2}{\alpha_{0}}\langle\omega, A\rangle+\frac{2 N}{\alpha_{0}} \int_{\Sigma} \sigma
$$

and we can choose

$$
\Gamma=\operatorname{Sup}_{(z, p) \in \Sigma \times V}\|\nu(z, p)\|_{(\tau, g)}, \quad \text { and } \quad N=\frac{\alpha_{0}}{2}+\frac{\Gamma^{4} \beta_{0}^{4}}{2 \alpha_{0}^{3}} .
$$

Therefore we can find a positive number

$$
\rho=\rho\left(\alpha_{0}, \beta_{0}, C_{0}, i(V, g), j, J, \nu, A, \sigma\right)
$$

such that

$$
\operatorname{Im}(\bar{u}) \subset \widehat{K}_{\rho}
$$

Projecting on $V$ we can complete the proof of Proposition 2.6. 
As pointed out in Introduction, generally speaking, on the noncompact manifold $V$ for a given $J \in \mathcal{J}_{\tau}^{m}(V, \omega)$ and an arbitrary small positive number

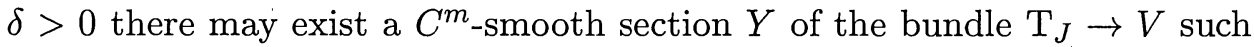
that $\|Y\|_{C^{m}}<\delta$, but $J \exp (-J Y) \notin \mathcal{J}_{\tau}^{m}(V, \omega)$. But for some noncompact symplectic manifolds we can prove:

Lemma 2.7. For a given $J_{0} \in \mathcal{J}_{\tau}^{m}(V, \omega)$, if there exist positive numbers $\alpha_{0}$, $\beta_{0}$ and an Riemann metric $g_{0}$ on $V$ such that

$$
\omega\left(\xi, J_{0} \xi\right) \geq \alpha_{0}\|\xi\|_{g_{0}}^{2}, \quad|\omega(\xi, \eta)| \leq \beta_{0}\|\xi\|_{g_{0}}\|\eta\|_{g_{0}}, \quad \text { for all } \xi, \eta \in T V
$$

then there exists a positive number $\delta_{0}$ such that

$$
\mathcal{U}_{\delta_{0}}^{m}\left(J_{0}\right)=\left\{J_{0} \exp \left(-J_{0} Y\right) \mid\|Y\|_{C^{m}} \leq \delta_{0}, Y \in C^{m}\left(\mathrm{~T}_{J_{0}}\right)\right\} \subset \mathcal{J}_{\tau}^{m}(V, \omega)
$$

for each integer $m \geq 1$. Here $\|\cdot\|_{C^{m}}$ is defined in terms of the covariant derivatives with respect to the Riemannian metric $g_{0}$. Furthermore, $\delta_{0}>0$ can be chosen so small that every $J \in \mathcal{U}_{\delta_{0}}^{m}\left(J_{0}\right)$ satisfies: $\omega(\xi, J \xi) \geq \frac{\alpha_{0}}{2}\|\xi\|_{g_{0}}^{2}$ for all $\xi \in T V$.

Proof. First note that the condition (10) imply that

$$
\frac{\alpha_{0}}{\beta_{0}}\|\xi\|_{g_{0}} \leq\left\|J_{0} \xi\right\|_{g_{0}} \leq \frac{\beta_{0}}{\alpha_{o}}\|\xi\|_{g_{0}}
$$

for all $\xi \in T V$. Specially, we have that $\alpha_{0} / \beta_{0} \leq\left\|J_{0}(p)\right\|_{g_{0}} \leq \beta_{0} / \alpha_{0}$ for all $p \in V$.

Next, for any $J=J_{0} \exp \left(-J_{0} Y\right) \in \mathcal{U}_{\delta_{0}}^{m}\left(J_{0}\right)$ and $p \in V, \xi \in T_{p} V$ we have

$$
\begin{aligned}
\omega(\xi, J(p) \xi) & =\omega\left(\xi, J_{0}(p) \exp \left(-J_{0}(p) Y(p)\right)\right) \\
& =\omega\left(\xi, J_{0}(p) \xi\right)+\omega\left(\xi, J_{0}(p)\left[\exp \left(-J_{0}(p) Y(p)\right)-I\right] \xi\right) \\
& =\omega\left(\xi, J_{0}(p) \xi\right)+\omega\left(\xi,\left[\exp \left(J_{0}(p) Y(p)\right)-I\right] J_{0}(p) \xi\right) \\
& \geq \alpha_{0}\|\xi\|_{g_{0}}^{2}-\beta_{0}\|\xi\|_{g_{0}}\left\|\left[\exp \left(J_{0}(p) Y(p)\right)-I\right] J_{0}(p) \xi\right\|_{g_{0}} \\
& \geq \alpha_{0}\|\xi\|_{g_{0}}^{2}-\frac{\beta_{0}^{2}}{\alpha_{0}}\|\xi\|_{g_{0}}^{2}\left\|\exp \left(J_{0}(p) Y(p)\right)-I\right\|_{g_{0}} .
\end{aligned}
$$


On the other hand, by (11) and the definition of exp

$$
\begin{aligned}
\left\|\exp \left(J_{0}(p) Y(p)\right)-I\right\|_{g_{0}} & \leq \sum_{k=1}^{\infty} \frac{\left\|J_{0}(p) Y(p)\right\|_{g_{0}}^{k}}{k !} \\
& \leq\left(\sum_{k=0}^{\infty} \frac{\left\|J_{0}(p) Y(p)\right\|_{g_{0}}^{k}}{k !}\right)\left\|J_{0}(p) Y(p)\right\|_{g_{0}} \\
& \leq\left\|J_{0}(p)\right\|_{g_{0}}\|Y(p)\|_{g_{0}} \exp \left(\left\|J_{0}(p)\right\|_{g_{0}}\|Y(p)\|_{g_{0}}\right) \\
& \leq \frac{\beta_{0}}{\alpha_{0}} \delta_{0} \cdot \exp \left(\frac{\beta_{0}}{\alpha_{0}} \delta_{0}\right) .
\end{aligned}
$$

Thus we get

$$
\omega(\xi, J(p) \xi) \geq\left[\alpha_{0}-\frac{\beta_{0}^{3} \delta_{0}}{\alpha_{0}} \exp \left(\frac{\beta_{0}}{\alpha_{0}} \delta_{0}\right)\right]\|\xi\|_{g_{0}}^{2} .
$$

Hence we can choose a positive number $\delta_{0} \leq \frac{1}{2}\left(\frac{\alpha_{0}}{\beta_{0}}\right)^{3}$ so small that $\alpha_{0}-$ $\frac{\beta_{0}^{3} \delta_{0}}{\alpha_{0}} \exp \left(\frac{\beta_{0}}{\alpha_{0}} \delta_{0}\right) \geq \alpha_{0} / 2$. Lemma 2.7 is proved.

Now every $\mathcal{U}_{\delta_{0}}^{m}\left(J_{0}\right)$ is a Banach manifold, but it is not separable or even has not a countable base. In order to be able to apply Sard-Smale theorem in the transversality arguments below we introduce the space of the following type, which is one of our key techniques in this paper.

Take a proper Morse function $h$ on $V$ and two sequences of regular values of it, $\mathrm{a}=\left\{a_{i}\right\}$ and $\mathrm{b}=\left\{b_{i}\right\}$ satisfying:

$$
\begin{aligned}
\min _{x \in V} h(x):=a_{1}<a_{2}<b_{1}<a_{3}<b_{2}<a_{4} & <\cdots \\
& <a_{k}<b_{k-1}<a_{k+1}<\cdots ;
\end{aligned}
$$

and denote $Q_{i}:=\left\{a_{i} \leq h \leq b_{i}\right\}, i=1, \ldots$, we have

$$
V=\bigcup_{i=1}^{\infty} Q_{i}, \quad Q_{i} \cap Q_{i+2}=\emptyset, \quad \operatorname{Int}\left(Q_{i}\right) \cap \operatorname{Int}\left(Q_{i+1}\right) \neq \emptyset, i=1, \ldots
$$

Moreover, every $Q_{i}$ is a smooth compact submanifold with smooth boundary and has the same dimension as $V$. Following [F] we may choose a sequence of sufficiently rapidly decreasing positive numbers $\varepsilon^{(i)}=\left\{\varepsilon_{k}^{(i)}\right\}_{k=1}^{\infty}$ such that the space $C_{\varepsilon^{(i)}}^{\infty}\left(\left.\mathrm{T}_{J_{0}}\right|_{Q_{i}}\right)$ of those smooth sections $X \in C^{\infty}\left(\mathrm{T}_{J_{0}} \mid Q_{i}\right)$ for which

$$
\|X\|_{\varepsilon^{(i)}}=\sum_{k=1}^{\infty} \varepsilon_{k}^{(i)}\|X\|_{C^{k}\left(Q_{i}\right)}<\infty
$$


is separable and dense in $L^{1}\left(\left.\mathrm{~T}_{J_{0}}\right|_{Q_{i}}\right)$. In addition we always require that all $\varepsilon_{1}^{(i)}$ equal to 1 . Let

$$
C_{\varepsilon^{(i)}}^{\infty}\left(\mathrm{T}_{J_{0}}^{(i)}\right):=\left\{X \in C^{\infty}\left(\mathrm{T}_{J_{0}}\right) \mid \operatorname{supp} X \subset Q_{i},\|X\|_{\varepsilon^{(i)}}<\infty\right\} .
$$

This is a separable Banach space with respect to norm $\|\cdot\|_{\varepsilon^{(i)}}$. We denote

$$
\mathcal{L}_{\varepsilon}\left(J_{0}, h, \mathrm{a}, \mathrm{b}\right)
$$

by the space of all sequences $\mathrm{X}=\left(X_{1}, X_{2}, \ldots\right)$ with $X_{i} \in C_{\varepsilon^{(i)}}^{\infty}\left(\mathrm{T}_{J_{0}}^{(i)}\right)$ and

$$
\|\mathrm{X}\|_{\varepsilon}=\sum_{i=1}^{\infty}\left\|X_{i}\right\|_{\varepsilon^{(i)}}<\infty
$$

Then $\left(\mathcal{L}_{\varepsilon}\left(J_{0}, h, \mathrm{a}, \mathrm{b}\right),\|\cdot\|_{\varepsilon}\right)$ is a separable Banach space. Let $\mathrm{B}\left(\mathcal{L}_{\varepsilon}\left(J_{0}\right.\right.$, $h, \mathrm{a}, \mathrm{b}) ; \delta)$ be a closed ball in this Banach space of radius $\delta$. Then, for sufficiently small $\delta \in\left(0, \delta_{0} / 2\right)$ that $\|\mathrm{X}\|_{\varepsilon} \leq \delta$ implies that $\left\|\sum_{i=1}^{\infty} X_{i}\right\|_{C^{1}} \leq 2 \delta$ and thus from Lemma 2.7 it follow that $J:=J_{0} \exp \left(-J_{0}\left(\sum_{i=1}^{\infty} X_{i}\right)\right)$ belongs to $\mathcal{J}_{\tau}(V, \omega)$ which is the space of all smooth $\omega$-tame almost complex structures, and $\left(V, \omega, J, g_{0}\right)$ is still $\mathrm{g}$. bounded. Later, we fix such a $\delta$ and for convenience denote by

$$
\Xi: \mathrm{B}\left(\mathcal{L}_{\varepsilon}\left(J_{0}, h, \mathrm{a}, \mathrm{b}\right) ; \delta\right) \rightarrow \mathcal{J}_{\tau}(V, \omega), \mathrm{X} \mapsto J_{0} \exp \left(-J_{0}\left(\sum_{i=1}^{\infty} X_{i}\right)\right) .
$$

and also by

$$
\mathcal{U}_{\delta}\left(J_{0}, h, \mathrm{a}, \mathrm{b}, \varepsilon\right)
$$

the image of $\mathrm{B}\left(\mathcal{L}_{\epsilon}\left(J_{0}, h, \mathrm{a}, \mathrm{b}\right) ; \delta\right)$ under $\Xi$. This set is not necessary connected in $\mathcal{J}_{\tau}(V, \omega)$.

Having the space many regularity results on compact symplectic manifolds can be generalized to noncompact geometrically bounded symplectic manifolds. In fact, the above construction can be suitably modified so that the result of the moduli spaces in [Mc1] may be generalized to any noncompact symplectic manifolds without boundary, that is, the following proposition holds.

Proposition 2.8. Given $A \in H_{2}(V)$ and a closed Riemann surface $\Sigma$ of genus $g$ with the complex structure $j$, and $J_{0}$ as above, then there is a subset 
$\mathrm{B}_{\text {reg }}\left(\mathcal{L}_{\varepsilon}\left(J_{0}, h, \mathrm{a}, \mathrm{b}\right) ; \delta\right)$ of the second category in $\mathrm{B}\left(\mathcal{L}_{\varepsilon}\left(J_{0}, h, \mathrm{a}, \mathrm{b}\right) ; \delta\right)$ such that for every $\mathrm{X} \in \mathrm{B}_{\text {reg }}\left(\mathcal{L}_{\varepsilon}\left(J_{0}, h, \mathrm{a}, \mathrm{b}\right) ; \delta\right)$ the space

$$
\mathcal{M}_{s}(\Sigma, A, \Xi(\mathrm{X}))
$$

of all simple $\Xi(\mathrm{X})$-holomorphic maps from $\Sigma$ to $V$ and representing $A$ is a smooth manifold of dimension $(1-g) \operatorname{dim} M+2 c_{1}(A)$ and with a natural orientation. Moreover, for any two $\mathrm{X}$ and $\mathrm{Y}$ in $\mathrm{B}_{\mathrm{reg}}\left(\mathcal{L}_{\varepsilon}\left(J_{0}, h, \mathrm{a}, \mathrm{b}\right) ; \delta\right)$ it may be proved that $\mathcal{M}_{s}(\Sigma, A, \Xi(\mathrm{X}))$ and $\mathcal{M}_{s}(\Sigma, A, \Xi(\mathrm{Y}))$ are oriented cobordant.

For every integer $m>1$ we denote by $\overline{H o m}_{J_{0}}^{m}(T \Sigma, T V)$ the space of the $C^{m}$-smooth sections of the bundle of anti- $J_{0}$-linear homomorphisms from $T \Sigma$ to $T V$ over $\Sigma \times V$. Consider the Banach vector bundle $\mathcal{H}^{m}$ over $\mathrm{B}\left(\mathcal{L}_{\varepsilon}\left(J_{0}, h, \mathrm{a}, \mathrm{b}\right) ; \delta\right)$ whose fibre at a point $\mathrm{X}$ is $\overline{\mathrm{Hom}}_{\Xi(\mathrm{X})}^{m}(T \Sigma, T V)$. It is easy to know that this is a separable $C^{m}$ Banach vector bundle. We call the elements of the bundle as inhomogeneous terms. Fix a large integer $m_{0}>0$ such that the conditions of the Sard-Smale theorem are satisfied. For every integer $m \geq m_{0}$ one may, as in [RT1], prove that there exists a subset $\mathcal{H}_{\text {reg }}^{m}$ of the second category in $\mathcal{H}^{m}$ such that for every $(\mathrm{X}, \nu) \in \mathcal{H}_{\text {reg }}^{m}$ the space

$$
\mathcal{M}_{A}^{m}(\Sigma, \Xi(\mathrm{X}), \nu)
$$

of all $(\Xi(\mathrm{X}), \nu)$-map from $\Sigma$ to $V$ representing $A$ is a $C^{m}$-smooth manifold of dimension $(1-g) \operatorname{dim} M+2 c_{1}(A)$ and with a natural orientation. Moreover, for any two pairs $(\mathrm{X}, \nu)$ and $(\mathrm{Y}, \mu)$ in $\mathcal{H}_{\text {reg }}^{m}$ it may be proved that $\mathcal{M}_{A}^{m}(\Sigma, \Xi(\mathrm{X}), \nu)$ and $\mathcal{M}_{A}^{m}(\Sigma, \Xi(\mathrm{Y}), \mu)$ are $C^{m}$ oriented cobordant. Specially, it should be noted that

$$
\mathcal{H}_{\text {reg }}^{m_{0}} \supseteq \mathcal{H}_{\text {reg }}^{m_{0}+1} \supseteq \ldots
$$

which implies that for any $(\mathrm{X}, \nu) \in \mathcal{H}_{\text {reg }}^{m}$ and $\left(\mathrm{X}^{\prime}, \nu^{\prime}\right) \in \mathcal{H}_{\text {reg }}^{m^{\prime}}$ with $m^{\prime}>m \geq$ $m_{0}$ the spaces $\mathcal{M}_{A}^{m}(\Sigma, \Xi(\mathrm{X}), \nu)$ and $\mathcal{M}_{A}^{m^{\prime}}\left(\Sigma, \Xi\left(\mathrm{X}^{\prime}\right), \nu^{\prime}\right)$ are also $C^{m}$ oriented cobordant.

Let $\mathcal{G}(V)$ be the set of all Riemann metrics on $V$ whose injectivity radius are more than zero and sectional curvatures have upper bounds. We also denote by $\mathcal{G J}_{\tau}(V, \omega)$ the set of all $J \in \mathcal{J}_{\tau}(V, \omega)$ which satisfy: $\omega(\xi, J \xi) \geq \alpha_{0}\|\xi\|_{g}^{2}$ and $|\omega(\xi, \eta)| \leq \beta_{0}\|\xi\|_{g}\|\xi\|_{g}$ for some fixed $g \in \mathcal{G}(V)$, constants $\alpha_{0}, \beta_{0}>0$ and all $\xi, \eta \in T V$. Obviously, for every g.bounded symplectic manifold $(V, \omega), \mathcal{G J}_{\tau}(V, \omega)$ is a nonempty open subset of $\mathcal{J}_{\tau}(V, \omega)$ with respect to $C^{1}$-topology. However, we do not affirm it to be connected. For every connected component $\mathcal{G}(V)_{c}$ of $\mathcal{G}(V)$ we denote $\mathcal{G J}_{\tau}(V, \omega)_{c}$ by 
the subset of $J \in \mathcal{G J}_{\tau}(V, \omega)$ for which $(V, \omega, J, g)$ is g.bounded for some $g \in \mathcal{G}(V)_{c}$. Using Bévennec's construction [ALP, p. 44] it is easily proved that every $\mathcal{G J}_{\tau}(V, \omega)_{c}$ is connected. Similarly, for every integer $m \geq m_{0}$ we also denote by

$$
\mathcal{G H}^{m}(V, \omega):=\left\{(J, \nu) \mid J \in \mathcal{G J}_{\tau}(V, \omega) \text { and } \nu \text { satifying(1) }\right\}
$$

and corresponding component $\mathcal{G H}^{m}(V, \omega)_{c}$, where $\nu \in \overline{\mathrm{Hom}}_{J}^{m}(T V, T M)$. Then the later is still connected.

\section{Transversality and Compactness.}

In this section we shall follow the methods in [RT1], [McSa1] to make arguments. Because the techniques are same basically we only give the necessary improvements and list the main results.

First of all, we start with the following notion. A pair $(\Sigma ; \overline{\mathbf{z}})$ of a connected Hausdorff topological space $\Sigma$ and $k$ different points $\overline{\mathbf{z}}=\left\{z_{1}, \ldots, z_{k}\right\}$ on it is called the semistable curve with $k$ marked points ([FO]) if there exists a finite family of smooth closed Riemann surfaces $\left\{\Sigma_{s}: s \in \Lambda\right\}$ and continuous maps $\pi_{\widetilde{\Sigma}_{s}}: \widetilde{\Sigma}_{s} \rightarrow \Sigma$ such that: $(i)$ each $\pi_{\widetilde{\Sigma}_{s}}$ is a local homeomorphism; (ii) for each $p \in \Sigma$ it holds that $1 \leq \sum_{s} \sharp \pi_{\widetilde{\Sigma}_{s}}^{-1}(p) \leq 2$, and all points which satisfy $\sum_{s} \sharp \pi_{\widetilde{\Sigma}_{s}}^{-1}(p)=2$ are isolated; (iii) for each $z_{i}, \sum_{s} \sharp \pi_{\tilde{\Sigma}_{s}}^{-1}\left(z_{i}\right)=1$. Denote by $\Sigma_{\text {sing }}:=\left\{p \in \Sigma: \sum_{s} \sharp \pi_{\widetilde{\Sigma}_{s}}^{-1}(p)=2\right\}$ the set of all singular points of $\Sigma$. Specially, each singular point $p$ such that $\sharp \pi_{\widetilde{\Sigma}_{s}}^{-1}(p)=2$ is called the self-intersecting point of $\Sigma$. Call $\Sigma_{s}:=\pi_{\widetilde{\Sigma}_{s}}\left(\widetilde{\Sigma}_{s}\right)$ the $s$-th components of $\Sigma$, and $\widetilde{\Sigma}_{s}$ the smooth resolution of $\Sigma_{s}$. Each $z_{i}$ is called the marked point. The points in $\pi_{\tilde{\Sigma}_{s}}^{-1}\left(\Sigma_{\text {sing }}\right)$ and $\pi_{\tilde{\Sigma}_{s}}^{-1}(\overline{\mathbf{z}})$ are called the singular points and the marked points on $\widetilde{\Sigma}_{s}$, respectively. Let $k_{s}$ be the number of all singular and marked points on $\widetilde{\Sigma}_{s}$ and $g_{s}$ be the genus of $\widetilde{\Sigma}_{s}$. The genus $g$ of $(\Sigma ; \overline{\mathbf{z}})$ is defined by

$$
1+\sum_{s} g_{s}+\sharp \operatorname{Inter}(\Sigma)-\sharp \operatorname{Comp}(\Sigma),
$$

where $\sharp \operatorname{Inter}(\Sigma)$ and $\sharp \operatorname{Comp}(\Sigma)$ stand for the number of the intersecting points on $\Sigma$ and that of the components of $\Sigma$ respectively.

If $k_{s}+2 g_{s} \geq 3$ we call the component $\left(\widetilde{\Sigma}_{s} ; \overline{\mathbf{z}}_{s}\right)$ stable. When all components of $(\Sigma ; \overline{\mathbf{z}})$ are stable we call $(\Sigma ; \overline{\mathbf{z}})$ the stable curve of genus $g$ and with $k$ marked points.

For the above genus $g$ stable curve $(\Sigma ; \overline{\mathbf{z}})$ a continuous map $f: \Sigma \rightarrow V$ is called $C^{l}(l \geq 1)$ if each $f \circ \pi_{\widetilde{\Sigma}_{s}}$ is so. The homology class of $f$ is defined 
by $f_{*}([\Sigma])=\sum_{s}\left(f \circ \pi_{\widetilde{\Sigma}_{s}}\right)_{*}\left(\left[\widetilde{\Sigma}_{s}\right]\right)$. An $C^{m}$ inhomogeneous term $\nu$ over $\Sigma$ is a set $\left\{\nu_{s}: s \in \Lambda\right\}$ of inhomogeneous terms, where each $\nu_{s}$ is an $C^{m}$ inhomogeneous term of $\widetilde{\Sigma}_{s}$ and they together satisfy the match conditions. A map $f: \Sigma \rightarrow V$ is called $(J, \nu)$-perturbed holomorphic if each $f \circ \pi_{\widetilde{\Sigma}_{s}}$ is $\left(J, \nu_{s}\right)$-perturbed holomorphic. Denote by $\mathcal{M}_{A}^{m}(\Sigma, J, \nu)$ the moduli space of all $(J, \nu)$-perturbed holomorphic maps from $\Sigma$ into $V$ with $f_{*}([\Sigma])=A$. Using the method in $\S 2$ and the arguments in [McSa1], [RT1, Prop. 4.13] it follows that for every given pair $\left(J^{\prime}, \nu^{\prime}\right)$ with $C^{m}$ inhomogeneous term $\nu^{\prime}$ there exists a pair $(J, \nu)$ with $C^{m}$ inhomogeneous term $\nu$ which may be arbitrarily close to it, such that moduli space $\mathcal{M}_{A}^{m}(\Sigma, J, \nu)$ is a $C^{m}$ smooth manifold of dimension $2 c_{1}(V)(A)+2 n(1-g)$. In order to get suitable compactification of the above moduli space the following form cusp-curve due to Gromov was introduced in [RT1]. Given a $k$-point genus $g$ stable curve $(\Sigma ; \overline{\mathbf{z}})$ as above, $\left(\Sigma^{\prime} ; \overline{\mathbf{z}}^{\prime}\right)$ is another $k$-point curve obtained from it as follows: First at some double points of $\Sigma$ we join chains of $\mathbb{C} P^{1}$ to separate the two components and then attach some trees of $\mathbb{C} P^{1}$, but require that if one attaches a tree of $\mathbb{C} P^{1}$ at a marked point $x_{i}$, this $x_{i}$ will be replaced by a point different from intersection points on some component of the tree, and under other cases the marked points do not change. The components of $\Sigma$ is called principal components and other bubble components. A continuous map $f: \Sigma^{\prime} \rightarrow(V, \omega)$ is called a $\Sigma$-cusp $(J, \nu)$-map if for each principal component $\Sigma_{s}$ the map $f \circ \pi_{\widetilde{\Sigma}_{s}}$ is $\left(J, \nu_{s}\right)$-perturbed holomorphic and the restriction of $f$ to a bubble component is a nonconstant $J$-holomorphic map. We define a $(\Sigma, J, \nu)$-cusp curve as an equivalence class of cusp maps modulo the parametrization groups of bubbles. Its homology class is defined as the sum of the homology classes of all components of the any cusp map representatives of it. Denote by $\mathcal{C M}_{A}^{m}(\Sigma, J, \nu)$ the set of all $(\Sigma, J, \nu)$-cusp curves with the total homology class $A$. For every element of the space one can obtain a reduced $(\Sigma, J, \nu)$-cusp curve by forgetting multiplicity of the multiple covering maps on bubble components and collapsing each subtree of the bubbles whose components have the same image. Notice that this new cusp curve may have different total homology class from the original one. We denote by $\overline{\mathcal{M}}_{A}^{m}(\Sigma, J, \nu)$ the set of all reduced $(\Sigma, J, \nu)$-cusp curve from $\mathcal{C M}_{A}^{m}(\Sigma, J, \nu)$. For the semi-positive closed symplectic manifold $(V, \omega)$ it was proved that $\mathcal{C M}_{A}^{m}(\Sigma, J, \nu)$ is the cusp curve compactification of $\mathcal{M}^{m}(\Sigma, J, \nu)$ and $\overline{\mathcal{M}}_{A}^{m}(\Sigma, J, \nu) \backslash \mathcal{M}^{m}(\Sigma, J, \nu)$ consists of finitely many strata and each stratum is also branchedly covered by a $C^{m}$-smooth manifold of codimension at least 2 ([RT1]). However, in our case $\mathcal{C M}_{A}^{m}(\Sigma, J, \nu)$ is only the closure of $\mathcal{M}^{m}(\Sigma, J, \nu)$ due to the noncompactness of $(V, \omega)$. In order to get desire results we assume that each $\nu_{s}$ in $\nu=\left\{\nu_{s}: s \in \Lambda\right\}$ satisfies (1). For any 
compact subset $K \subset V$ let $\mathcal{C M}_{A}^{m}(\Sigma, J, \nu, K)$ be the subset of $\mathcal{C M}_{A}^{m}(\Sigma, J, \nu)$ consisting of all elements whose images are intersecting with $K$. Then we have

Proposition 3.1. Let $(V, \omega, g, J)$ be a g.bounded symplectic manifold and $(\Sigma ; \overline{\mathbf{z}})$ a $k$-point genus $g$ stable curve with a bounded inhomogeneous term $\nu$ over it. Then there exists a positive number $\eta=\eta\left(A, i(V, g), C_{0}, \alpha_{0}, \beta_{0}, \nu\right)$ such that

$$
\bigcup_{f \in \mathcal{C M}_{A}^{m}(\Sigma, J, \nu, K)} \operatorname{Im}(f) \subset K_{\eta} .
$$

In fact, if $\Sigma_{1}, \ldots, \Sigma_{p}$ are principal components of $\Sigma^{\prime}$ which only depend on $\Sigma$, and $B_{1}, \ldots, B_{l}$ are bubble components of $\Sigma^{\prime}$ then it follows from the proof of Lemma 4.5 in [RT1] that there is a uniform constant $c$ such that $E\left(f_{\Sigma_{i}}\right) \leq c\left(\omega\left(f_{*}\left(\left[\Sigma_{i}\right]\right)\right)+1\right), E\left(f_{B_{j}}\right) \leq c \omega\left(f_{*}\left(\left[B_{j}\right]\right)\right)$ and therefore

$$
\sum_{i=1}^{p} E\left(f_{\Sigma_{i}}\right)+\sum_{j=1}^{l} b_{j} E\left(f_{B_{j}}\right) \leq c(\omega(A)+p) .
$$

Here the positive integer $b_{1}, \ldots, b_{l}$ satisfy $A=\sum_{i=1}^{p} f_{*}\left(\left[\Sigma_{i}\right]\right)+$ $\sum_{j=1}^{l} b_{j} f_{*}\left(\left[B_{j}\right]\right)$. These show that one can find a positive integer $l_{0}=$ $l_{0}(\omega(A), \Sigma, V, \omega, K)$ such that it bounds $l$ uniformly. Moreover, for given area forms $\sigma_{s}$ on $\widetilde{\Sigma}_{s}(s=1, \ldots, p)$ one can find a sufficiently large $N>0$ such that all $\left(\widetilde{\Sigma}_{s} \times V, \tilde{\omega}_{s}, \tau_{s} \oplus g, \tilde{J}_{s}\right)$ are g.bounded. Here $\tilde{\omega}_{s}=N \sigma_{s} \times \omega$ and $\tilde{J}_{s}$ are defined as in Lemma 2.5. From the proof of Proposition 2.6 it follows that $\omega\left(f_{*}\left(\left[\Sigma_{s}\right]\right)\right) \geq-N \int_{\Sigma_{s}} \sigma_{s}$ for each $s$. Combing these with $\sum_{s=1}^{p} \omega\left(f_{*}\left(\left[\Sigma_{s}\right]\right)\right) \leq \omega(A)$ we get that $\omega\left(f_{*}\left(\left[\Sigma_{s}\right]\right)\right) \leq \omega(A)+p N \min _{s} \int_{\Sigma_{s}} \sigma_{s}$ and $\omega\left(f_{*}\left(\left[B_{j}\right]\right)\right) \leq p \omega(A)+p(p-1) N \min _{s} \int_{\Sigma_{s}} \sigma_{s}$. Now since $\Sigma^{\prime}$ is connected, by repeatedly using Lemma 2.5 we can finish the proof of Proposition 3.1. As a consequence of this proposition and Proposition 3.1 in [RT1] we have

Corollary 3.2. For any compact subset $K \subset V, \mathcal{C M}_{A}^{m}(\Sigma, J, \nu, K)$ is compact.

As in [RT1, §4], $\overline{\mathcal{M}}_{A}^{m}(\Sigma, J, \nu) \backslash \mathcal{M}_{A}^{m}(\Sigma, J, \nu)$ can be stratified and their strata are indexed by $\mathcal{D}_{A, \Sigma}^{J, \nu}$ (cf. [RT1] for definition). For a compact subset $K \subset V$ we denote by $\mathcal{D}_{A, \Sigma}^{J, \nu}(K)$ the subset of $\mathcal{D}_{A, \Sigma}^{J, \nu}$ consisting of those $D \subset$ $\mathcal{D}_{A, \Sigma}^{J, \nu}$ which has a $\Sigma$-cusp $(J, \nu)$-map representative intersecting with $K$. Then carefully checking the proof of Lemma 4.5 in [RT1] we can prove 
Lemma 3.3. For any compact subsets $K \subset V, \mathcal{D}_{A, \Sigma}^{J, \nu}(K)$ is a finite set. But $\mathcal{D}_{A, \Sigma}^{J, \nu}$ may be a countable set.

Corresponding to Theorem 4.2 in [RT1] we may use the argument method in $\S 2$ to get the following structure theorem.

Theorem 3.4. Let $(V, \omega)$ be a g.bounded semi-positive symplectic manifold, then there is a dense subset $\mathcal{G H}_{\text {reg }}^{m}(V, \omega)$ in $\mathcal{G} \mathcal{H}^{m}(V, \omega)$ such that for each pair $(J, \nu) \in \mathcal{G} \mathcal{H}_{\text {reg }}^{m}(V, \omega)$, the complementary $\overline{\mathcal{M}}_{A}^{m}(\Sigma, J, \nu) \backslash \mathcal{M}_{A}^{m}(\Sigma, J, \nu)$ consists of at most countable many strata and each stratum is branchedly covered by a $C^{m}$-smooth manifold of codimension at least 2. Moreover, there are only finitely many strata of $\overline{\mathcal{M}}_{A}^{m}(\Sigma, J, \nu) \backslash \mathcal{M}_{A}^{m}(\Sigma, J, \nu)$ which can intersect with $\overline{\mathcal{M}}_{A}^{m}(\Sigma, J, \nu, K)$ for every compact subset $K \subset V$.

More precisely, if for each $D \in \mathcal{D}_{A, \Sigma}^{J, \nu}$ we denote by $\mathcal{M}_{\Sigma}^{m}(D, J, \nu)$ the space of all $C^{m}$-smooth $(\Sigma, J, \nu)$-cusp curves such that the homeomorphism type of its domain, homology class of each component, components which have the same image are specified by $D$. Then from Theorem 4.7 and Proposition 4.14 in [RT1] and the arguments in $\S 2$ we can obtain

Theorem 3.5. For every $(J, \nu)$ in a dense subset $\mathcal{G H}_{\text {reg }}^{m}(V, \omega)$ of $\mathcal{G H}^{m}(V, \omega)$ and a $D$ in $\mathcal{D}_{A, \Sigma}^{J, \nu}$ there exists a $C^{m}$-smooth branched covering manifold $\mathcal{N}_{\Sigma}^{m}(\bar{D}, J, \nu)$ of $\mathcal{M}_{\Sigma}^{m}(D, J, \nu)$ whose dimension is not more than $2 c_{1}(V)(A)+$ $2 n(1-g)-2 k_{D}-2 s_{D}$. Here $k_{D}$ is the number of bubble components of $D$ and $s_{D}$ is the number of marked points which are bubbling points. Moreover, for any two pairs $(J, \nu)$ and $\left(J^{\prime}, \nu^{\prime}\right)$ in $\mathcal{G H}_{\mathrm{reg}}^{m}(V, \omega) \cap \mathcal{G H}_{\mathrm{reg}}(V, \omega)_{c}$ there is a path $\left(J_{\tau}, \nu_{\tau}\right)$ connecting $(J, \nu)$ and $\left(J^{\prime}, \nu^{\prime}\right)$ in $\mathcal{G H}_{\mathrm{reg}}^{m}(V, \omega)_{c}$ such that $\cup_{t \in[0,1]} \mathcal{N}_{\Sigma}^{m}\left(\bar{D}, J_{\tau}, \nu_{\tau}\right) \times\{t\}$ is a $C^{m}$-smooth cobordism.

It should be noted that the manifolds $\mathcal{M}_{A}^{m}(\Sigma, J, \nu)$ and $\mathcal{N}_{\Sigma}^{m}(\bar{D}, J, \nu)$ carry a canonical orientation.

Denote by $\mathcal{B D}_{A, \Sigma}^{J, \nu}$ the subset of $\mathcal{D}_{A, \Sigma}^{J, \nu}$ whose elements contain the bubble components. From Theorem 3.5 we have

$$
\overline{\mathcal{M}}_{A}^{m}(\Sigma, J, \nu) \backslash \mathcal{M}_{A}^{m}(\Sigma, J, \nu) \subset \bigcup_{D \in \mathcal{B D}_{A, \Sigma}^{J, \nu}} \mathcal{M}_{\Sigma}^{m}(D, J, \nu)
$$

\section{Gromov-Witten Invariants.}

In this section we shall follow the method in [McSa1] to define the GromovWitten invariants of Ruan-Tian's form-mixed invariants. First of all, we 
recall some evaluation maps. For a $k$-point genus $g$ stable curve $(\Sigma, \overline{\mathbf{z}})$, $\overline{\mathbf{z}}=\left(z_{1}, \ldots, z_{k}\right)$ and integers $l>0$ consider the $C^{m}$-smooth map

$$
e_{(\Sigma, \overline{\mathbf{z}}, J, \nu)}^{m}: \mathcal{M}_{A}^{m}(\Sigma, J, \nu) \times \Sigma^{l} \mapsto V^{k} \times V^{l}=V^{k+l}
$$

given by

$$
\left(f ; y_{1}, \ldots, y_{l}\right) \mapsto\left(f\left(z_{1}\right), \ldots, f\left(z_{k}\right) ; f\left(y_{1}\right), \ldots, f\left(y_{l}\right)\right) .
$$

For each $D \in \mathcal{B D}_{A, \Sigma}^{J, \nu}$ the similar map

$$
e_{(D, J, \nu)}^{m}: \mathcal{M}_{\Sigma}^{m}(D, J, \nu) \times\left(\Sigma^{\prime}\right)^{l} \mapsto V^{k+l}
$$

can be defined. For each $D \in \mathcal{B D}_{A, \Sigma}^{J, \nu}$, let $\pi_{D}^{m}: \mathcal{N}_{\Sigma}^{m}(\bar{D}, J, \nu) \rightarrow \mathcal{M}_{\Sigma}^{m}(D, J, \nu)$ be a branched covering defined below Definition 4.6 in [RT1]. The composition maps $e_{D}^{m}=e_{(D, J, \nu)}^{m} \circ \pi_{D}^{m}$ satisfy

$$
\bigcap_{S \subset \mathcal{M}_{A}^{m}(\Sigma, J, \nu) \times \Sigma^{l} \text { compact }} \overline{e_{(\Sigma, \overline{\mathbf{z}}, J, \nu)}^{m}\left(\left[\mathcal{M}_{A}^{m}(\Sigma, J, \nu) \times \Sigma^{l}\right] \backslash S\right)} \subset \bigcup_{D \in \mathcal{B D}_{A, \Sigma}^{J, \nu}} \operatorname{Im}\left(e_{D}^{m}\right) .
$$

These show that $e_{(\Sigma, \overline{\mathbf{z}}, J, \nu)}^{m}$ is a $C^{m}$-smooth pseudo-cycle. Let us recall the notion of the pseudo-cycles introduced on the page 90 of [McSa1]. A $k$ dimensional $C^{m}$-smooth pseudo-cycle in $V$ is a $C^{m}$-smooth map $f: M \rightarrow$ $V$ defined on an oriented $C^{m}$-smooth $k$-dimensional manifold $M$ (possibly noncompact) such that the boundary

$$
f\left(M^{\infty}\right)=\bigcap_{S \subset M \text { compact }} \overline{f(M-S)}
$$

of $f(M)$ is of dimension at most $k-2$, i.e., there exists a $C^{m}$-smooth manifold $W$ of dimension at most $k-2$ and a $C^{m}$-smooth map $g: W \rightarrow V$ such that $f\left(M^{\infty}\right) \subset g(W)$. Furthermore, if $\overline{f(M)}$ is also compact in $V$ then we call $f$ as strong pseudo-cycle. Clearly, in a compact manifold these two notions are equivalent. According to the definition the identity map $V \rightarrow V$ is not a strong pseudo-cycle in the noncompact manifold $V$. From Remark 7.1.1 in [McSa1] it easily follows that every integral homology class $\alpha \in H_{2}(V, \mathbb{Z})$ can be represented by a $C^{\infty}$ strong pseudo-cycle $f: M \rightarrow V$. Every strong pseudo-cycle determines a homology class, and bordant pseudo-cycles determine the same homology class. But in the noncompact manifold $V$ a pseudo-cycle does not necessarily determine a homology class as the identity map from $V$ to $V$. Moreover, it is easily checked 
that the product of two (strong) pseudo-cycles is also a (strong) pseudocycle in the product manifold. If $f_{k}: M \rightarrow V_{k}$ are (strong) pseudo-cycles, $k=1,2$, then the map $M \rightarrow V_{1} \times V_{2}, m \mapsto\left(f_{1}(m), f_{2}(m)\right)$ is also (strong) pseudo-cycle. In $\S 5$ below we will need these conclusions. Two pseudo-cycles $e: P \rightarrow V$ and $f: Q \rightarrow V$ are called transverse if either $e(P) \cap f(Q)=\emptyset$ or $e\left(P^{\infty}\right) \cap \overline{f(Q)}=\emptyset, \overline{e(P)} \cap f\left(Q^{\infty}\right)=\emptyset$ and $T_{x} V=\operatorname{Imde}(p)+\operatorname{Imd} f(q)$ whenever $e(p)=f(q)=x$. However, it should be noted that for two transverse pseudo-cycles $e$ and $f$ as above, if one of them is a strong pseudo-cycle $\Delta(e, f):=\{(p, q) \in P \times Q \mid e(p)=f(q)\}$ is a compact manifold of dimension $\operatorname{dim} P+\operatorname{dim} Q-\operatorname{dim} V$. This statement can be derived from the definition of transversality of pseudo-cycles directly. Specially,it is a finite set if $P$ and $Q$ are of complementary dimension. Under our case Lemma 7.1.2 in [McSa1] are not applicable due to the noncompactness of the manifold $V$, which implies that $\operatorname{Diff}^{r}(V)$ is not separable Banach manifold for every integer $r>0$. We must give its suitable modification form. This can be obtained with our method in $\S 2$.

Fix a large integer $r>0$ and as in (12) we denote by

$$
\chi_{i}^{r}:=\left\{X \in \chi^{r}(V) \mid \operatorname{supp} X \subset Q_{i},\|X\|_{C^{r}}<\infty\right\},
$$

where $\chi^{r}(V)$ are the space of all $C^{r}$-vector fields on $V$, and

$$
\|X\|_{C^{r}}=\sup _{x \in V}|X(x)|_{g}+\sup _{x \in V}\left|\nabla_{g} X(x)\right|_{g}+\cdots+\sup _{x \in V}\left|\nabla_{g}^{r} X(x)\right|_{g},
$$

$\nabla_{g}$ is the Levi-Civita connection of metric $g$. Then every $\left(\chi_{i}^{r},\|X\|_{C^{r}}\right)$ is separable Banach space. Denote by

$$
\chi^{r}(V)_{0}
$$

the space of all sequences $\mathrm{X}=\left(X_{1}, X_{2}, \ldots\right)$ with $X_{i} \in \chi_{i}^{r}$ and

$$
\|\mathrm{X}\|_{g r}=\sum_{k=1}^{\infty}\left\|X_{k}\right\|_{C^{r}}<\infty .
$$

Then it is easily proved that $\left(\chi^{r}(V)_{0},\|\cdot\|_{g r}\right)$ is a separable Banach space. Note that every $\mathrm{X} \in \chi^{r}(V)_{0}$ determines a bounded $C^{r}$-smooth vector field, denoted by $\rho_{r}(\mathrm{X})=\sum_{i=1}^{\infty} X_{i}$. Clearly, the image of $\rho_{r}$ contains all smooth vector fields with compact support on $V$. But every $C^{r}$-smooth bounded vector field on complete Riemann manifolds can uniquely determine a oneparameter $C^{r}$-smooth diffeomorphism group. Let us denote by $\left\{F_{t}(\rho(\mathrm{X}))\right.$ : $t \in \mathbb{R}\}$ the group determined by $\rho_{r}(\mathrm{X})$. Define

$$
\mathcal{F}^{r}: \chi^{r}(V)_{0} \rightarrow \operatorname{Diff}^{r}(V), \mathrm{X} \mapsto F_{1}\left(\rho_{r}(\mathrm{X})\right) .
$$


It is easily checked that $\mathcal{F}^{r}$ is a $C^{r}$-smooth map. Corresponding to Lemma 7.1.2 in [McSa1] we have the following lemma.

Lemma 4.1. If a $C^{p}$-smooth pseudo-cycle e $: P \rightarrow V$ and $a C^{q}$-smooth one $f: Q \rightarrow V$ satisfy

$$
\operatorname{dim} P+\operatorname{dim} Q \geq \operatorname{dim} V
$$

then

(i) for every sufficiently large integer $r>\min \{p, q\}$ there exists a set $\chi^{r}(V, e, f) \subset \chi^{r}(V)_{0}$ of the second category such that $e$ is transverse to $\mathcal{F}^{r}(\mathrm{X}) \circ f$ for all $\mathrm{X} \in \chi^{r}(V, e, f)$; these $\chi^{r}(V, e, f)$ also satisfy:

$$
\chi^{r}(V, e, f) \supseteq \chi^{r+1}(V, e, f) \supseteq \ldots,
$$

which implies that for any $\mathrm{X} \in \chi^{r}(V, e, f)$ and $\mathrm{Y} \in \chi^{s}(V, e, f)$ with $s>r$ it holds that

$$
\left(\mathcal{F}^{r}(\mathrm{X}) \circ f\right) \cdot e=\left(\mathcal{F}^{s}(\mathrm{Y}) \circ f\right) \cdot e
$$

provided that the equality in (27) also holds and one of $f$ and $e$ is a strong pseudo-cycle;

(ii) if the equality in (27) holds, $e$ and $f$ are transverse and one of them is a strong pseudo-cycle, then $\Delta(e, f)$ is a finite set and in this case we denote by $\nu(x, y)$ the intersection number of e and $f$ at $(x, y) \in \Delta(e, f)$, and define

$$
e \cdot f=\sum_{(x, y) \in \Delta(e, f)} \nu(x, y)
$$

(iii) the intersection number $e \cdot f$ depends only on the bordism classes of $e$ and $f$ when one of them varies in the bordism class of the strong pseudo-cycle.

Proof. The proof can be finished as in [McSa1]. We only need prove that the map

$$
\Theta: P \times Q \times \chi^{r}(V)_{0} \rightarrow V \times V:(p, q, \mathrm{X}) \mapsto\left(e(p), \mathcal{F}^{r}(\mathrm{X})(f(q))\right)
$$

is transverse to the diagonal $\triangle_{V}$. For any $(p, q, \mathrm{X}) \in \Theta^{-1}\left(\triangle_{V}\right)$ the differential of $\Theta$ at it is given by

$$
\begin{aligned}
& D \Theta(p, q, \mathrm{X})(\xi, \eta, \mathrm{Y}) \\
& =\left(D e(p)(\xi), D\left(\mathcal{F}^{r}(\mathrm{X}) \circ f\right)(q)(\eta)+\left[D \mathcal{F}^{r}(\mathrm{X})(\mathrm{Y})\right](f(q))\right),
\end{aligned}
$$


where $(\xi, \eta, \mathrm{Y}) \in T_{p} P \times T_{q} Q \times \chi^{r}(V)_{0}$. Let $m=e(p)=\mathcal{F}^{r}(\mathrm{X})(f(q))$. For any given $(u, v) \in T_{(m, m)}(V \times V)$ we wish to find $w \in T_{m} V$ and $(\xi, \eta, \mathrm{Y}) \in$ $T_{p} P \times T_{q} Q \times \chi^{r}(V)_{0}$ such that

$$
\begin{gathered}
D e(p)(\xi)=w+u \\
D\left(\mathcal{F}^{r}(\mathrm{X}) \circ f\right)(q)(\eta)+\left[D \mathcal{F}^{r}(\mathrm{X})(\mathrm{Y})\right](f(q))=w+v .
\end{gathered}
$$

By taking $\xi=0, \eta=0$ and $w=-u$ we need only find $\mathrm{Y} \in \chi^{r}(V)_{0}$ such that

$$
\left[D \mathcal{F}^{r}(\mathrm{X})(\mathrm{Y})\right](f(q))=v-u .
$$

For $f(q)=\left[\mathcal{F}^{r}(\mathrm{X})\right]^{-1}(m)$, by definition of $\mathcal{F}^{r}(\mathrm{X})$, it is $\alpha_{\mathrm{X}}(1)$, where $\alpha_{\mathrm{X}}(t)$ is the unique solution of the initial value problem

$$
\dot{\alpha}_{\mathrm{X}}(t)=\left(\sum_{i=1}^{\infty} X_{i}\right)\left(\alpha_{\mathrm{X}}(t)\right), \quad \alpha_{\mathrm{X}}(0)=f(q) .
$$

For $s \in(-1,1)$ and $\mathrm{Y} \in \chi^{r}(V)_{0}$ we denote by $\alpha_{\mathrm{X}+s \mathrm{Y}}(t)$ the unique solution of the initial problem

$$
\dot{\alpha}_{\mathrm{X}+s \mathrm{Y}}(t)=\left(\sum_{i=1}^{\infty} X_{i}+s Y_{i}\right)\left(\alpha_{\mathrm{X}+s \mathrm{Y}}(t)\right), \quad \alpha_{\mathrm{X}+s \mathrm{Y}}(0)=f(q) .
$$

Then we need to find $\mathrm{Y} \in \chi^{r}(V)_{0}$ such that

$$
\left.\frac{d}{d s} \alpha_{\mathrm{X}+s \mathrm{Y}}(1)\right|_{s=0}=v-u \text {. }
$$

By localization method it is easy to find a smooth vector field $Z$ with compact support on $V$ such that for the unique solution curve family $\beta\left(\rho_{r}(\mathrm{X})+s Z\right)(t)$ of $\rho_{r}(\mathrm{X})+s Z$ with initial value $f(q)$ at zero it holds that

$$
\left.\frac{d}{d s} \beta\left(\rho_{r}(\mathrm{X})+s Z\right)(1)\right|_{s=0}=v-u .
$$

Now using the unit decomposition technique it is easy to find a $\mathrm{Y} \in \chi^{r}(V)_{0}$ with $Z=\rho_{r}(\mathrm{Y})$. Thus we prove the transversality.

Moreover, the standard computation shows that the restriction of the natural projection $\Pi$ from $P \times Q \times \chi^{r}(V)_{0}$ to $\chi^{r}(V)_{0}$ to $\Theta^{-1}\left(\triangle_{V}\right)$ is a Fredholm operator with index

$$
\text { Index }\left(\Pi \mid \Theta^{-1}\left(\triangle_{V}\right)\right)=\operatorname{dim} P+\operatorname{dim} Q-\operatorname{dim} V,
$$


which is only dependent on the dimension of $P, Q$ and $V$. Under our assumption this index is less than or equal to zero. Thus we need only fix an integer $r>0$ such that Sard-Smale theorem can be applied. The remainder of the arguments are the same as that in [McSa1].

Now let a $k$-point genus $g$ stable curve $(\Sigma, \overline{\mathbf{z}}), A \in H_{2}(V, \mathbb{Z})$ and the pair $(J, \nu)$ satisfy the regularity requirements in $\S 2$ and $\S 3$. The integral homology classes $\left\{\alpha_{i}\right\}_{1 \leq i \leq k}$ and $\left\{\beta_{j}\right\}_{1 \leq j \leq l}$ of $V$ satisfy

$$
\sum_{1}^{k}\left(2 n-\operatorname{deg}\left(\alpha_{i}\right)\right)+\sum_{1}^{l}\left(2 n-\operatorname{deg}\left(\beta_{j}\right)-2\right)=2 c_{1}(V)(A)+2 n(1-g)
$$

We choose strong pseudo-cycles $f_{i}: P_{i}: \rightarrow V$ and $h_{j}: Q_{j} \rightarrow V$ representing $\alpha_{i}$ and $\beta_{j}(1 \leq i \leq k, 1 \leq j \leq l)$, respectively. Then

$$
f:=\prod_{i=1}^{k} f_{i} \times \prod_{j=1}^{l} h_{j}: \prod_{i=1}^{k} P_{i} \times \prod_{j=1}^{l} Q_{j} \rightarrow V^{k+l}
$$

is a strong pseudo-cycle representing the integral homology class $\prod_{i} \alpha_{i} \times$ $\prod_{j} \beta_{j} \in H_{*}\left(V^{k+l}, \mathbb{Z}\right)$. Since the compositions $f \circ \phi$ of this $f$ with any $\phi \in \operatorname{Diff}^{r}\left(V^{k+l}\right)$ are also $C^{r}$-strong pseudo-cycles representing the same class, using Lemma 4.1 we can assume that $f$ is transverse to $e_{(\Sigma, \overline{\mathbf{z}}, J, \nu)}^{m}$ and all $e_{(D, J, \nu)}^{m}$ because of the countability of $\mathcal{B D}_{A, \Sigma}^{J, \nu}$. By Lemma 4.1 and (36) we can define the mixed invariant

$$
\Phi_{(A, \omega, g)}\left(\alpha_{1}, \ldots, \alpha_{k} \mid \beta_{1}, \ldots, \beta_{l}\right)=f \cdot e_{(\Sigma, \overline{\mathbf{z}}, J, \nu)}^{m} .
$$

In the case that (36) does not hold we also define

$$
\Phi_{(A, \omega, g)}\left(\alpha_{1}, \ldots, \alpha_{k} \mid \beta_{1}, \ldots, \beta_{l}\right)=0 .
$$

As in [RT1] we can use the arguments in $\S 2$ and $\S 3$ to prove that $\Phi_{(A, \omega, g)}\left(\alpha_{1}, \ldots, \alpha_{k} \mid \beta_{1}, \ldots, \beta_{l}\right)$ is independent of choices of $(J, \nu)$ in a dense subset of $\mathcal{H J}^{m}(V, \omega)_{c}$, marked points $z_{1}, \ldots, z_{k}$ in $\Sigma$, the conformal structures on $\Sigma$, sufficiently large integers $r, m$ and strong pseudo-cycles $\left(P_{i}, f_{i}\right),\left(Q_{j}, h_{j}\right)$ representing $\alpha_{i}, \beta_{j}$ for a given component $\mathcal{H J}^{m}(V, \omega)_{c}$ of $\mathcal{H J}^{m}(V, \omega)$. For two different components we do not know what relationships there are between corresponding invariants. When talking about some property of the invariants we always mean them to be with respect to some fixed component without special statements. Similarly, the corresponding 
results to Proposition 2.4, 2.5 and 2.6, 2.7 in [RT1] can be proved. In particular, under our assumptions one can define the invariant $\Phi_{(A, \omega, \mathcal{C})}$ as $\S 7$ in [RT1] and prove the composition law:

$$
\Phi_{(A, \omega, g)}\left(\alpha_{1}, \ldots, \alpha_{k} \mid \beta_{1}, \ldots, \beta_{l}\right)=\Phi_{(A, \omega, \mathcal{C})}\left(\alpha_{1}, \ldots, \alpha_{k} \mid \beta_{1}, \ldots, \beta_{l}\right)
$$

where $\mathcal{C}=(\Sigma, \overline{\mathbf{z}})$ is a $k$-point genus $g$ stable curve and $\alpha_{1}, \ldots, \alpha_{k}, \beta_{1}, \ldots, \beta_{l}$ are integral homology classes of $V$.

As to the deformation invariance of these invariants with respect to the semi-positive deformation class of $\omega$ we introduce the following notion of deformation equivalence. Two semi-positive symplectic form $\omega_{0}$ and $\omega_{1}$ on a (noncompact) geometrically bounded symplectic manifold $V$ is called $d e-$ formedly equivalent if there exists a smooth 1-parameter family of semipositive symplectic forms $\omega_{t}$ connecting $\omega_{0}$ and $\omega_{1}$, and a family of almost complex structures $J_{t}$ such that all $\left(V, \omega_{t}, J_{t}, g\right)$ are uniformly geometrically bounded with respect to some metric $g \in \mathcal{G}(V)$, that is, there exist constants $\alpha_{0}$ and $\beta_{0}$ such that two inequalities in $1^{\circ}$ of Definition 2.3 hold uniformly for all $\omega_{t}$. As usual we may use the above method to prove our Gromov-Witten invariants are invariant under such semi-positive deformations of $\omega$.

Example 4.2. For any closed manifold $N$ and any closed 2-form $\Omega$ on $N$ consider the symplectic manifold $(M, \omega)=\left(T^{*} N, \omega_{\text {can }}+\pi^{*} \Omega\right)$ then for any $k$-point genus $g$ stable curve $(\Sigma, \overline{\mathrm{z}}), A \in H_{2}(V, \mathbb{Z})$, the integral homology classes $\left\{\alpha_{i}\right\}_{1 \leq i \leq k}$ and $\left\{\beta_{j}\right\}_{1 \leq j \leq l}$ of $V$ we have

$$
\Phi_{(A, \omega, g)}\left(\alpha_{1}, \ldots, \alpha_{k} \mid \beta_{1}, \ldots, \beta_{l}\right)=0 .
$$

In fact, take any Riemannian metric $h$ on $N$ and denote by $H$ the induced Riemannian metric on $T^{*} N$ by $h$. Then from proof of Proposition 4.1 in [Lu2] it easily follows that all symplectic manifolds $\left(T^{*} N, \omega_{t}\right)$ are uniformly geometrically bounded with respect to $H$. Here $\omega_{t}=\omega_{\text {can }}+t \Omega$, $t \in[0,1]$. Furthermore, the proof there also shows that one can take a smooth family of almost complex structures $J_{t}$ such that every $J_{t}$ is $\omega_{t^{-}}$ compatible and $\left(M, \omega_{t}, J_{t}, H\right)$ are uniformly geometrically bounded. Now Chern class $c_{1}\left(T M, J_{t}\right)$ is independent of $t$ and thus they are all zero because $c_{1}\left(T M, J_{0}\right)=0$ is clear. Hence the symplectic forms $\omega_{0}=\omega_{\text {can }}$ and $\omega_{1}$ are deformedly equivalent. But it is clear that $\Phi_{\left(A, \omega_{\text {can }}, g\right)}\left(\alpha_{1}, \ldots, \alpha_{k} \mid \beta_{1}, \ldots, \beta_{l}\right)$ always vanishes. The above deformation invariance leads to the conclusion.

In order to define the quantum homology ${ }^{2}$ of $(V, \omega)$ we need to assume

\footnotetext{
${ }^{2} \mathrm{P}$. Seidel pointed out that in the orginal version using the Poincare duality on noncompact manifolds does not give rise to a product on $H_{c}^{*}(V)$, and should consider the quantum homology of $(V, \omega)$ instead.
} 
that

$$
\Gamma_{\omega}=H_{2}^{S}(V, \mathbb{Z}) / H_{2}^{S}(V, \mathbb{Z})_{0} \text { is finitely generated, }
$$

so that the Novikov ring $\Lambda_{\omega}$ associated to the homomorphism $\omega: \Gamma_{\omega} \rightarrow \mathbb{R}$ is well-defined. Here $H_{2}^{S}(V, \mathbb{Z})_{0}$ is the subgroup of classes $\alpha$ in $H_{2}^{S}(V, \mathbb{Z})$ such that $\langle[\omega], \alpha\rangle=0$ and $\left\langle c_{1}(V, \omega), \alpha\right\rangle=0$. As usual we denote by

$$
Q H_{*}(V)=H_{*}(V) \otimes \Lambda_{\omega}
$$

where $H_{*}(V)$ stand for of $H_{*}(V, \mathbb{Z})$ modulo torsion. The quantum intersect product is given by

$$
\alpha *_{V} \beta=\sum_{A \in \Gamma_{\omega}}\left(\alpha *_{V} \beta\right)_{A} \otimes e^{A} \in Q H_{k+l-2 n}(V)
$$

for $\alpha \in H_{k}(V)$ and $\beta \in H_{l}(V)$. Here $\left(\alpha *_{V} \beta\right)_{A} \in H_{k+l+2 c_{1}(A)-2 n}(V)$ is determined by

$$
\left(\alpha *_{V} \beta\right)_{A} \cdot V \gamma=\Phi_{(A, \omega, 0)}(\alpha, \beta, \gamma) \text { for all } \gamma \in H_{*}(V) .
$$

This gives an ring structure on $Q H_{*}(V)$.

Remark 4.3. For given integral homology classes $\alpha_{1}, \ldots, \alpha_{k}, \beta_{1}, \ldots, \beta_{l}$ and their strong pseudo-cycles representatives $f_{i}: P_{i} \rightarrow V, h_{j}: Q_{j} \rightarrow V$ as in (37) it follows from $V$ being noncompact g.bounded that there exist the diffeomorphisms $\phi \in \operatorname{Diff}^{r}(V)$ such that the images of $f$ and $\hat{\phi} \circ f:=\prod_{i}^{k} \phi \circ$ $f_{i} \times \prod_{j}^{l} \phi \circ h_{j}$ are not intersecting each other and even have the larger distances. But $\hat{\phi} \circ f$ and $f$ are representing the same homology classes, therefore from our results that if their Gromov-Witten invariants are not zero then the maps in $\mathcal{M}_{A}(\Sigma, J, \nu)$ are distributed over $V$ in an even way. In the same time this seems also to show the complexity of the distributions of the holomorphic curves in the general noncompact symplectic manifolds.

\section{Gromov-Witten Invariants of Compact Symplectic Manifolds with Contact Type Boundary.}

Let $(V, \omega)$ is a $2 n$-dimensional compact symplectic manifold with contact type boundary $\partial V$. That is, there is a one-form $\alpha$ on $\partial V$ such that $d \alpha=\left.\omega\right|_{\partial V}$ and $\alpha \wedge(d \alpha)^{n-1}$ is a volume form on $\partial V$. Such a form $\alpha$ is called a contact 
form on $\partial V$. One can associate a noncompact symplectic manifold $(\widetilde{V}, \widetilde{\omega})$ as follows:

$$
\widetilde{V}=V \bigcup_{\partial V \times\{1\}} \partial V \times[1,+\infty), \quad \widetilde{\omega}= \begin{cases}\omega & \text { on } V \\ d(t \alpha) & \text { on } \partial V \times[1,+\infty) .\end{cases}
$$

Here $t$ is the second coordinate. For a $J \in \mathcal{J}(V, \omega)$ and a Riemannian metric $h$ on $V$ we may extend them to $\widetilde{J}$ and $\widetilde{h}$ respectively so that $\widetilde{J}$ and $\widetilde{h}$ are constant on the $\partial V \times\{t\}$. It is easily checked that $(\widetilde{V}, \widetilde{\omega}, \widetilde{J}, \widetilde{h})$ is a g. bounded symplectic manifold. Moreover the inclusion $i: V \rightarrow \widetilde{V}$ induces clear isomorphisms $i_{*}: H_{*}(V, \mathbb{Z}) \rightarrow H_{*}(\widetilde{V}, \mathbb{Z})$ and $i^{*}: H^{*}(\widetilde{V}, \mathbb{Z}) \rightarrow H^{*}(V, \mathbb{Z})$. It is clear that $i^{*}\left(c_{1}(\widetilde{V}, \widetilde{J})\right)=c_{1}(V, J)$ and $i^{*}([\widetilde{\omega}])=[\omega]$. Consequently, $(\widetilde{V}, \widetilde{\omega})$ is semi-positive if only and if $(V, \omega)$ is semi-positive. For a class $\alpha \in H_{*}(V, \mathbb{Z})$ we denote $\widetilde{\alpha}$ by $i_{*}(\alpha)$. Then for a given $k$-point genus $g$ stable curve $(\Sigma, \bar{z}), A \in H_{2}(V)$ and integral homology classes $\left\{\alpha_{i}\right\}_{1 \leq i \leq k}$ and $\left\{\beta_{j}\right\}_{1 \leq j \leq l}$ of $V$ satisfying (36) we define

$$
\Phi_{(A, \omega, g)}\left(\alpha_{1}, \ldots, \alpha_{k} \mid \beta_{1}, \ldots, \beta_{l}\right):=\Phi_{(\tilde{A}, \widetilde{\omega}, g)}\left(\widetilde{\alpha}_{1}, \ldots, \widetilde{\alpha}_{k} \mid \widetilde{\beta}_{1}, \ldots, \widetilde{\beta}_{l}\right) .
$$

Since both the space of all Riemannian metrics on $V$ and $\mathcal{J}(V, \omega)$ are contractible it is easy to check that the left of (42) is independent on the choices of $J$ in a dense subset of $\mathcal{J}(V, \omega)$, marked points $z_{1}, \ldots, z_{k}$ in $\Sigma$ and conformal structures on $\Sigma$. Moreover, they also satisfy the axioms that GromovWitten invariants satisfy on closed symplectic manifolds. Notice that $(\widetilde{V}, \widetilde{\omega})$ always satisfies the assumption in (41). One may naturally define a quantum ring $Q H_{*}(V)=H_{*}(V) \otimes \Lambda_{\omega}$ from (42) and the agruments above Remark 4.3 .

\section{Rigidity of the Loops in the Group of Hamiltonian Diffeomorphisms with Compact Support.}

The quantum homology had been used to study the topology of symplectomorphism groups and Hamiltonian symplectomorphism groups on closed symplectic manifolds in [Se1], [Le], [LMP]. In this section we will use the techniques developed in the previous sections and their ideas to study these groups on noncompact $\mathrm{g}$. bounded symplectic manifolds. Without special statements our $2 n$-dimensional symplectic manifold $(V, \omega)$ is always assumed to satisfy the following condition:

$$
A \in \pi_{2}(V), 2-n \leq c_{1}(A)<0 \Longrightarrow \omega(A) \leq 0 .
$$


Given an element $\phi \in \pi_{1}(\operatorname{Diff}(V), i d)$ and any a loop $S^{1} \rightarrow \operatorname{Diff}(V), t \mapsto \phi_{t}$ representing it one can define an endomorphism $\partial_{\phi}: H_{*}(V, \mathbb{Q}) \rightarrow H_{*+1}(V, \mathbb{Q})$ by setting $\partial_{\phi}([C])$ as a homology class represented by the cycle $S^{1} \times C \rightarrow$ $V,(t, x) \mapsto \phi_{t}(x)$ for a cycle $C$ in $V$. The main result in [LMP] is that for a loop $\phi$ in the group $\operatorname{Ham}(V, \omega)$ the endomorphism $\partial_{\phi}$ vanishes identically if a $2 n$-dimensional closed symplectic manifold $(V, \omega)$ satisfies (43). In this section we generalize their result as follows:

Theorem 6.1. If a $2 n$-dimensional g.bounded symplectic manifold $(V, \omega)$ satisfies (41), (43) then for any loop $\phi$ in $\operatorname{Ham}^{c}(V, \omega)^{3}$ the endomorphism $\partial_{\phi}$ vanishes.

Let $G S(V)$ be the set of the symplectic structures $\omega$ on $V$ satisfying (41), (43). For any $\omega \in G S(V)$ we denote by $S_{\omega}: \pi_{1}\left(\operatorname{Symp}_{0}^{c}(V, \omega)\right) \rightarrow$ $\pi_{1}(\operatorname{Diff}(V), i d)$ and $H_{\omega}: \pi_{1}\left(\operatorname{Ham}_{0}^{c}(V, \omega)\right) \rightarrow \pi_{1}(\operatorname{Diff}(V), i d)$ the homomorphisms induced by the group inclusions respectively. As in [LMP], as a consequence of Theorem 6.1 we get the following result on the rigidity of Hamiltonian loops.

Corollary 6.2. For an element $\phi$ in $\pi_{1}(\operatorname{Diff}(V), i d)$ if there exist $\omega_{1}$ and $\omega_{2}$ in $G S(V)$ such that $\phi \in \operatorname{Im}\left(H_{\omega_{1}}\right) \cap \operatorname{Im}\left(S_{\omega_{2}}\right)$ then it also belongs to $\operatorname{Im}\left(H_{\omega_{2}}\right)$.

For a $2 n$-dimensional compact smooth manifold $M$ with nonempty boundary $\partial M$ we denote $\operatorname{Cont}(M)$ by the set of all symplectic structures on it for which (43) holds and $\partial M$ is of contact type. Diff $(M, \partial M)$ denote the subgroup consisting of all elements $F \in \operatorname{Diff}(M)$ whose restriction to $\partial M$ is the identity. For a symplectic structure $\omega$ on $M$ we denote by the subgroups

$$
\begin{aligned}
\operatorname{Symp}(M, \partial M, \omega) & :=\operatorname{Diff}(M, \partial M) \cap \operatorname{Symp}(M, \omega), \\
\operatorname{Ham}(M, \partial M, \omega) & :=\operatorname{Diff}(M, \partial M) \cap \operatorname{Symp}(M, \omega) .
\end{aligned}
$$

By [Se2] these spaces may have infinitely many connected components. Notice that in Exercise 10.13 on the page 318 of [McSa2] it was pointed out that for a noncompact symplectic manifold $(V, \omega)$ without boundary the flux homomorphism is still well-defined on $\widetilde{\operatorname{Symp}}_{0}(V, \omega)$ and the corresponding result to Theorem 10.12 also holds when $\operatorname{Symp}_{0}(V, \omega)$ is replaced by $\operatorname{Symp}_{0}^{c}(V, \omega)$. In fact, carefully checking the proof Theorem 10.12 in [McSa2] one can get the stronger conclusion that for the isotopy

$$
[0,1] \rightarrow \operatorname{Symp}_{0}^{c}(V, \omega), t \mapsto \psi_{t}
$$

\footnotetext{
${ }^{3} \mathrm{P}$. Seidel had constructed an example with a nontrival Hamiltonian loop with compact support.
} 
with $\psi_{0}=i d$ and $\operatorname{Flux}\left(\left\{\psi_{t}\right\}\right)=0$ one actually make it to be isotopic with fixed endpoints to a Hamiltonian isotopy $\left\{\phi_{t}\right\}$ such that the support does not increase. That is, if a compact subset $K \subset V$ is such that $\operatorname{Supp} \psi_{t} \subseteq K$ for all $t \in[0,1]$, then $\left\{\phi_{t}\right\}$ may be required to satisfy: Supp $\phi_{t} \subseteq K, \forall t \in[0,1]$. Using this remark and Corollary 6.2 we may obtain

Corollary 6.3. For a 2n-dimensional compact smooth manifold $M$ with nonempty boundary $\partial M$ and $\phi \in \pi_{1}(\operatorname{Diff}(M, \partial M)$, id $)$ if $\operatorname{Cont}(M)$ is nonempty then for any two $\omega_{1}$ and $\omega_{2}$ in $\operatorname{Cont}(M)$ it holds that $\phi \in$ $\operatorname{Im}\left(H_{\omega_{1}}\right) \cap \operatorname{Im}\left(S_{\omega_{2}}\right)$ if and only if $\phi \in \operatorname{Im}\left(H_{\omega_{2}}\right) \cap \operatorname{Im}\left(S_{\omega_{1}}\right)$.

In fact, let $\left(\widetilde{M}, \widetilde{\omega}_{1}\right)$ and $\left(\widetilde{M}, \widetilde{\omega}_{2}\right)$ be the symplectic expansion as made in $\S 5$ they obviously satisfy (41) (43). Moreover, every element of $\operatorname{Diff}_{0}(M, \partial M)$ can be extended into one of $\operatorname{Diff}_{0}^{c}(\widetilde{M})$ by the identity extension. Thus $\operatorname{Symp}_{0}\left(M, \partial M, \omega_{i}\right)$ and $\operatorname{Ham}_{0}\left(M, \partial M, \omega_{i}\right)$ may be viewed as the subgroups of $\operatorname{Symp}_{0}^{c}\left(\widetilde{M}, \widetilde{\omega}_{i}\right)$ and $\operatorname{Ham}_{0}^{c}\left(\widetilde{M}, \widetilde{\omega}_{i}\right), i=1,2$, respectively. Now the conclusion may be derived from Corollary 6.2.

As pointed out in [LMP] their results may be generalized to arbitrary closed symplectic manifolds with the methods developed in [FO], [LT1, LT2], [R3], [Sie]. However, as done in the previous sections it seem to be very hard to generalize our results to arbitrary noncompact g.bounded symplectic manifolds with their methods.

It is well-known that there exists a one-to-one correspondence between elements of $\pi_{1}(\operatorname{Symp}(V, \omega))$ and isomorphism classes of symplectic fibre bundles over $S^{2}$ with fibre $(V, \omega)$ (cf. [LMP], [Se1]). For a given loop $\phi_{t \in[0,1]}$ in $\operatorname{Symp}(V, \omega)$ the correspondent symplectic fibre bundle $P_{\phi} \rightarrow S^{2}$ may be obtained as follows: let $D^{+}$and $D^{-}$be two copies of the closed disk $D^{2}$ of radius 1 of the plane bounded by $S^{1}$, one can glue the trivial fibre bundles $D^{ \pm} \times(V, \omega)$ by a map $\Phi: \partial D^{+} \times V \rightarrow \partial D^{-} \times V:(2 \pi t, x) \mapsto\left(-2 \pi t, \phi_{t}(x)\right)$. According to [Se1] a symplectic fibre bundle with fibre $(V, \omega)$ on $S^{2}$ is a smooth fibre bundle $\pi: E \rightarrow S^{2}$ together with a smooth family $\Omega=\left(\Omega_{b}\right)_{b \in S^{2}}$ of symplectic forms on its fibres satisfying locally trivial condition and the transition function taking its value in the group $\operatorname{Symp}(V, \omega)$. He also call a symplectic fibre bundle $(E, \Omega) \rightarrow S^{2}$ as Hamiltonian if there is a closed two-form $\widetilde{\Omega}$ on $E$ such that $\widetilde{\Omega} \mid E_{b}=\Omega_{b}$ for all $b \in S^{2}$. Later, we call such a closed two-form $\widetilde{\Omega}$ on the Hamiltonian fibre bundle as Hamiltonian form.

Furthermore, from proof of Proposition 10.17 on the page 320 of [McSa2] one can prove that for every loop $S^{1} \rightarrow \operatorname{Ham}^{c}(V, \omega), t \mapsto \phi_{t}$ there is a smooth function $H_{\phi}: S^{1} \times V \rightarrow$ with compact support to generate it. Especially, 
there is an exact sequence of groups

$$
0 \rightarrow \pi_{1}\left(\operatorname{Ham}^{c}(V, \omega)\right) \rightarrow \pi_{1}\left(\operatorname{Symp}_{0}^{c}(V, \omega)\right) \stackrel{\text { Flux }}{\rightarrow} H_{c}^{1}(V, \mathbb{R}),
$$

where Flux is the flux homomorphism. Consequently, from the proof of Proposition 2.9 in [Se1] it follows that for a loop $\phi$ in $\operatorname{Symp}^{c}(V, \omega)$ the symplectic fibre bundle $P_{\phi} \rightarrow S^{2}$ is Hamiltonian if and only if the loop $\phi$ may be homotopic to a Hamiltonian loop in $\operatorname{Symp}^{c}(V, \omega)$, that is, a loop in $\operatorname{Ham}^{c}(V, \omega)$.

As in [LMP] using the Wang exact sequence of pair $\left(P_{\phi}, S^{2}\right)$ :

$$
\cdots \rightarrow H_{q-1}(V, \mathbb{Z}) \stackrel{\partial_{\phi_{*}}}{\rightarrow} H_{q}(V, \mathbb{Z}) \stackrel{i_{*}}{\rightarrow} H_{q}\left(P_{\phi}, \mathbb{Z}\right) \rightarrow H_{q-2}(V, \mathbb{Z}) \rightarrow \cdots
$$

the proof of Theorem 6.1 can be reduced to the following equivalent theorem.

Theorem 6.4. Let $(V, \omega)$ be as in Theorem 6.1 and $\phi$ a loop in $\operatorname{Ham}^{c}(V, \omega)$. Then the homomorphism $i: H_{*}(V, \mathbb{Q}) \rightarrow H_{*}\left(P_{\phi}, \mathbb{Q}\right)$ is injective.

In order to prove this theorem we need to give the detailed construction in Proposition 2.9 of [Se1] since the more conclusions are needed. Let $D_{1 / 3}^{+}=$ $\left\{z \in D^{+}|1 / 3 \leq| z \mid \leq 1\right\}$ and $D_{1 / 3}^{-}=\left\{z \in D^{-}|1 / 3 \leq| z \mid \leq 1\right\}$. Denote by $(r, t)_{ \pm}$the polar coordinate in $D^{ \pm}$with $t \in S^{1}=\mathbb{R} / \mathbb{Z}$. In the set $\Delta:=$ $\left\{(r, t)_{+},(r, t)_{-} \mid(r, t) \in D\right\}$ we define an equivalence relation $\sim$ as follows: the equivalence class of $(r, t)_{+}$is $[r, t]_{+}=\left\{(r, t)_{+},(-r+5 / 3,-t)_{-}\right\}=[-r+$ $5 / 3,-t]_{-}$if $2 / 3 \leq r \leq 1$, those of $(r, t)_{+}$and $(r, t)_{-}$are $[r, t]_{+}$and $[r, t]_{-}$ respectively if $0 \leq r<2 / 3$. Then $S^{2}=\Delta / \sim$ and $U_{ \pm}:=\left\{[r, t]_{ \pm} \mid(r, t) \in D\right\}$ form an open cover of $S^{2}$. $U_{+} \cap U_{-}=\left\{[r, t]_{+}=[-r+5 / 3,-t]_{-} \mid(r, t) \in\right.$ $\left.[2 / 3,1] \times S^{1}\right\}$. The coordinate charts $\varphi_{ \pm}: D \rightarrow U_{ \pm},(r, t) \mapsto[r, t]_{ \pm}$give an atlas on $S^{2}$. The transition map is:

$\varphi_{-}^{-1} \circ \varphi_{+}: D_{1 / 3}:=\{z \in D|2 / 3 \leq| z \mid \leq 1\} \rightarrow D_{1 / 3},(r, t) \rightarrow(-r+5 / 3,-t)$.

We also consider the formal set

$$
\left\{\left((r, t)_{ \pm}, x\right) \mid(r, t, x) \in D \times V\right\}
$$

and in it we define an equivalence relation $\sim \phi$ as follows: the equivalence class of $\left((r, t)_{+}, x\right)$ is $[r, t, x]_{+}^{\phi}=\left\{\left((r, t)_{+}, x\right)\right\}$ if $0 \leq r<2 / 3$, that of $\left((r, t)_{-}, x\right)$ is $[r, t, x]_{-}^{\phi}=\left\{\left((r, t)_{-}, x\right)\right\}$ if $0 \leq r<2 / 3$, and that of $\left((r, t)_{+}, x\right)$ is $[r, t, x]_{+}^{\phi}=\left[-r+5 / 3,-t, \phi_{t}(x)\right]_{-}^{\phi}:=\left\{\left((r, t)_{+}, x\right),\left((-r+5 / 3,-t)_{-}, \phi_{t}(x)\right)\right\}$ if $2 / 3 \leq r \leq 1$. Then the set, denoted by $P_{\phi}$, of all equivalence classes of 
elements in the set of (44) is a symplectic fibre bundle with fibre $(V, \omega)$. Two bundle charts $\Phi_{+}: U_{+} \times V \rightarrow P_{\phi} \mid U_{+},\left([r, t]_{+}, x\right) \mapsto[r, t, x]_{+}^{\phi}$ and $\Phi_{-}: U_{-} \times V \rightarrow P_{\phi} \mid U_{-},\left([r, t]_{-}, x\right) \mapsto[r, t, x]_{-}^{\phi}$ form an bundle atlas of $P_{\phi}$. The transition map is given by

$$
\begin{aligned}
\Phi_{-}^{-1} \circ \Phi_{+}: U_{+} \cap U_{-} \times V \rightarrow U_{+} \cap U_{-} \times V & ,\left([r, t]_{+}, x\right) \\
& \mapsto\left([-r+5 / 3,-t]_{-}, \phi_{t}(x)\right) .
\end{aligned}
$$

Denote by $p_{ \pm}: U_{ \pm} \times V \rightarrow V$ the natural projections to the second factor, and $\omega^{ \pm}:=p_{ \pm}^{*} \omega$. Define a one-form $\theta_{\phi}$ on $U_{+} \times V$ as follows: $\theta_{\phi}\left(\left([r, t]_{+}, x\right)\right)=$ $-\delta(r) H_{\phi}\left(t, \phi_{t}(x)\right) d t$. Here $H_{\phi}: S^{1} \times V \rightarrow \mathbb{R}$ is a smooth function generating $\phi_{t \in[0,1]}$ and having compact support, $\delta:[0,1] \rightarrow[0,1]$ is a monotone smooth function such that $\delta(r)=0$ for $0 \leq r \leq 1 / 4$ and $\delta(r)=r$ for $1 / 3 \leq r \leq 1$. In this paper we always fix this $\delta$ function. Clearly, $\theta_{\phi}$ has compact support. Straightforward computation shows that the closed two-forms $\left(\Phi_{+}^{-1}\right)^{*}\left(\omega^{+}+\right.$ $\left.d \theta_{\phi}\right)$ and $\left(\Phi_{-}^{-1}\right)^{*} \omega^{-}$are the same on overlap $\left.P_{\phi}\right|_{U_{+}} \cap U_{-}$. Thus they define a closed two-form $\widetilde{\Omega}_{\phi}$ on $P_{\phi}$ by

$$
\left.\widetilde{\Omega}_{\phi}\right|_{P_{\phi} \mid U_{+}}=\left(\Phi_{+}^{-1}\right)^{*}\left(\omega^{+}+d \theta_{\phi}\right) \quad \text { and }\left.\quad \widetilde{\Omega}_{\phi}\right|_{P_{\phi} \mid U_{-}}=\left(\Phi_{-}^{-1}\right)^{*} \omega^{-} .
$$

Let a compact subset $K \subseteq V$ be such that $\operatorname{Supp} H_{\phi} \subseteq S^{1} \times K$. Then from the above definition it easily follows that

$$
P_{\phi} \backslash\left(\Phi_{+}\left(U_{+} \times K\right) \bigcup \Phi_{-}\left(U_{-} \times K\right)\right)=S^{2} \times(V \backslash K),
$$

and on the set of (47) it holds that

$$
\widetilde{\Omega}_{\phi}=p_{2}^{*} \omega
$$

where $p_{2}: S^{2} \times V \rightarrow V$ is the natural projection. Moreover, one can easily prove that the above two-form $\widetilde{\Omega}_{\phi}$ is a Hamiltonian form on $P_{\phi}$ and also satisfies:

$$
\pi_{*} \widetilde{\Omega}_{\phi}^{n+1}=0 \quad \text { on } S^{2} \backslash\left\{[r, t]_{+} \in S^{2} \mid 1 / 4<r<1 / 3\right\},
$$

where $\pi_{*}$ is the fiber integration map, and $\Omega_{\phi}$ a smooth family of symplectic forms on the fibres of $P_{\phi} \rightarrow S^{2}$. Different from the case that $V$ is the closed symplectic manifold we neither know the existence of a Hamiltonian form $\widetilde{\Omega}$ on $P_{\phi}$ such that $\pi_{*} \widetilde{\Omega}^{n+1}=0$ nor the uniqueness of such forms if they exist. A Hamiltonian form $\widetilde{\Omega}$ on $P_{\phi}$ is called to have CS property if there are 
compact subsets $K_{\phi} \subset V$ and $\widehat{K}_{\phi} \subset P_{\phi}$ such that $P_{\phi} \backslash \widehat{K}_{\phi}=S^{2} \times\left(V \backslash K_{\phi}\right)$ and on them it holds that $\widetilde{\Omega}=p_{2}^{*} \omega$. Let us denote by

$$
\mathcal{H}(\phi)=\mathcal{H}\left(P_{\phi}\right)
$$

the set of all Hamiltonian forms having CS property on $P_{\phi}$. Since for any two Hamiltonian fibre bundles $P_{\phi}$ and $P_{\psi}$ on $S^{2}$ obtained from loops $\phi_{t \in[0,1]}$ and $\psi_{t \in[0,1]}$ in $\operatorname{Ham}_{0}^{c}(V, \omega)$ one can always find compact subsets $K \subset V$, $\widehat{K}_{\phi} \subset P_{\phi}$ and $\widehat{K}_{\psi} \subset P_{\psi}$ such that

$$
P_{\phi} \backslash \widehat{K}_{\phi}=P_{\psi} \backslash \widehat{K}_{\psi}=S^{2} \times(V \backslash K),
$$

we may say a symplectic fibre bundle isomorphism $\mathrm{I}^{\phi \psi}$ between $P_{\phi}$ and $P_{\psi}$ to have CS property if it is the identity map on the sets in (50), that is, $\mathrm{I}^{\phi \psi}(z, v)=(z, v)$ for all $(z, v) \in S^{2} \times(V \backslash K)$. Clearly, such an isomorphism induces a natural bijection $\mathrm{I}^{\phi \psi *}$ from $\mathcal{H}(\psi)$ to $\mathcal{H}(\phi)$ by the pull-back map.

For every $\widetilde{\Omega} \in \mathcal{H}(\phi)$ and the standard symplectic form $\sigma$ on $S^{2}$ it is easily proved that there is always a large constant $c(\widetilde{\Omega}, \phi)>0$ such that all two-forms $\widetilde{\Omega}+c \pi^{*} \sigma$ are symplectic forms on $P_{\phi}$ for all $c \geq c_{\phi}$. Though these symplectic forms are also the Hamiltonian form on $P_{\psi}$, but they have no CS property.

Given a Hamiltonian form $\widetilde{\Omega}$ on $P_{\phi}$, in [Se1] two continuous sections $s_{0}$ and $s_{1}$ of $P_{\phi}$ are called $\Gamma_{\omega}$-equivalent if $\widetilde{\Omega}_{\phi}\left(s_{0}\right)=\widetilde{\Omega}_{\phi}\left(s_{1}\right)$ and $c_{1}\left(T P_{\phi}^{\text {vert }}\right)\left(s_{0}\right)=c_{1}\left(T P_{\phi}^{\text {vert }}\right)\left(s_{1}\right)$. The key point is this definition being independent of the choice of $\widetilde{\Omega}$ (cf. [Se1]).

Following [Se1] we denote by $\mathcal{J}\left(P_{\phi}, \Omega_{\phi}\right)$ the space of smooth families $\mathbf{J}=\left(J_{z}\right)_{z \in S^{2}}$ of almost complex structures on the fibre of $P_{\phi}$ such that $J_{z}$ is $\Omega_{\phi z}$-compatible for all $z$. In other words, $\mathbf{J}$ is a smooth section of a bundle over $S^{2}$ whose fibre at a point $z \in S^{2}$ is the space $\mathcal{J}\left(P_{\phi z}, \Omega_{\phi z}\right)$. For the positively oriented complex structure $j$ on $S^{2}$ and $\mathbf{J} \in \mathcal{J}\left(P_{\phi}, \Omega_{\phi}\right), \widehat{\mathcal{J}}(j, \mathbf{J})$ denote the space of all almost complex structures $\hat{J}$ on $P_{\phi}$ compatible with $j$ and $\mathbf{J}$, that is, $\hat{J}$ satisfying: $D \pi \circ \hat{J}=j \circ D \pi$ and $\hat{J} \mid P_{\phi z}=J_{z}$ for all $z \in S^{2}$. Similarly, for every integer $m \geq 1$ we denote $\widehat{\mathcal{J}}^{m}(j, \mathbf{J})$ by the space of all $C^{m}$ smooth almost complex structures on $P_{\phi}$ satisfying the above conditions. A smooth section $s: S^{2} \rightarrow P_{\phi}$ is called $(j, \hat{J})$-holomorphic if $d s \circ j=\hat{J} \circ d s$. For a given $\widetilde{\Omega} \in \mathcal{H}(\phi)$, from the above arguments it is not difficult to prove that all symplectic manifolds $\left(P_{\phi}, \widetilde{\Omega}+c \pi^{*} \sigma\right)$ are g.bounded with respect to some $\hat{J} \in \widehat{\mathcal{J}}(j, \mathbf{J})$ and some Riemannian metric on $P_{\phi}$. To see this point we choose a $g \in \mathcal{G}(V)$. Let $\tau_{0}$ be the standard metric on $S^{2}$. Notice that the above arguments show that one can choose a Riemannian metric $G$ on $P_{\phi}$ 
such that it equals to $\tau_{0} \oplus g$ outside a compact subset. When $g$ takes over a connected component $\mathcal{G}(V)_{c}$ of $\mathcal{G}(V)$ all corresponding Riemannian metrics on $P_{\phi}$ also form a connected subset of all Riemannian metrics on $P_{\phi}$, denoted by $\mathcal{G}\left(P_{\phi}\right)_{c}$. Later we always fix a component without special statements. For a $G \in \mathcal{G}\left(P_{\phi}\right)_{c}$ we denote $G_{z}$ by the induced metric on fibre $P_{\phi z}$ then one can use the standard method to find $\mathbf{J} \in \mathcal{J}\left(P_{\phi}, \Omega_{\phi}\right)$ such that the family of symplectic manifolds $\left\{\left(P_{\phi z}, \Omega_{\phi z}, G_{z}\right)\right\}_{z \in S^{2}}$ is uniformly g.bounded. That is, their sectional curvature has a uniform upper bound, the injectivity radius has a uniform positive lower bound and there exist positive constants $\alpha_{0}$ and $\beta_{0}$ such that

$$
\begin{aligned}
\Omega_{\phi z}\left(\xi, J_{z} \xi\right) & \geq \alpha_{0}\|\xi\|_{G_{z}}^{2} \quad \text { and } \\
\left|\Omega_{\phi z}(\xi, \eta)\right| & \leq \beta_{0}\|\xi\|_{G_{z}}\|\eta\|_{G_{z}}, \forall z \in S^{2}, \xi, \eta \in T P_{\phi z}
\end{aligned}
$$

We denote by $\mathcal{G} \mathcal{J}\left(P_{\phi}, \Omega_{\phi}\right)_{c}$ all such $\mathbf{J} \in \mathcal{J}\left(P_{\phi}, \Omega_{\phi}\right)$ constructed from $\Omega_{\phi}$ and some $G \in \mathcal{G}\left(P_{\phi}\right)_{c}$ with the standard method. On the other hand from $\left(\widetilde{\Omega}+c \pi^{*} \sigma\right) \mid P_{\phi z}=\Omega_{\phi z}$ and $G \mid P_{\phi z}=G_{z}$ it follows that the almost complex structure $\hat{J}$ on $P_{\phi}$ constructed from $G$ and $\widetilde{\Omega}+c \pi^{*} \sigma$ with the standard method must be in $\widehat{\mathcal{J}}(j, \mathbf{J})$ and such that $\left(P_{\phi}, \widetilde{\Omega}+c \pi^{*} \sigma, \hat{J}, G\right)$ is also g.bounded. Now fix such a $\mathbf{J} \in \mathcal{J}\left(P_{\phi}, \Omega_{\phi}\right)$ and a $\hat{J} \in \widehat{\mathcal{J}}(j, \mathbf{J})$, and as in $\S 2$ we can construct a separable Banach space so that the transversity arguments in $\S 7$ of [Se1] can be completed in our case. That is, under our assumptions, we can find $\hat{J} \in \widehat{\mathcal{J}}(j, \mathbf{J})$ such that

(i) $\left(P_{\phi}, \widetilde{\Omega}+c \pi^{*} \sigma, \hat{J}, G\right)$ is g.bounded,

(ii) the space $\mathcal{S}\left(P_{\phi}, \Omega_{\phi}, j, \hat{J}, D\right)$ of all $(j, \hat{J})$-holomorphic sections of $P_{\phi}$ representing a $\Gamma_{\omega}$-equivalence class $D$ of a section of $P_{\phi}$ is a smooth manifold of dimension $2 n+2 c_{1}\left(T P_{\phi}^{\text {vert }}\right)(D)$ and for chosen two different points $z_{1}, z_{2} \in S^{2}$ in advance and isomorphisms $F_{k}^{\phi}:\left(P_{\phi z_{k}}, \Omega_{\phi z_{k}}\right) \rightarrow$ $(V, \omega), k=1,2$, the maps

$$
\mathrm{EV}_{k}^{\phi D}: \mathcal{S}\left(P_{\phi}, \Omega_{\phi}, j, \hat{J}, D\right) \rightarrow V, \quad s \mapsto F_{k}^{\phi}\left(s\left(z_{k}\right)\right)
$$

are pseudo-cycles in the sense of $\S 7.1$ of [McSa1].

Later we will fix such a $\hat{J}$ and a $c \geq c_{\phi}$ without special statements. For two integral homology classes $\alpha, \beta \in H_{*}(V, \mathbb{Z})$ and their strong pseduocycles representatives $f_{M}: M \rightarrow V$ and $f_{N}: N \rightarrow V$ we can, as in $\S 4$, show that there exist $H \in \operatorname{Diff}(V \times V)$ such that the pseduo-cycle $\mathrm{EV}^{\phi D}:=$ 
$\left(\mathrm{EV}_{1}^{\phi D}, \mathrm{EV}_{2}^{\phi D}\right)$ and strong pseudo-cycle $H \circ\left(f_{M} \times f_{N}\right)$ transversely intersect provided that

$$
2 n+2 c_{1}\left(T P_{\phi}^{\text {vert }}\right)(D)+\operatorname{deg}(\alpha)+\operatorname{deg}(\beta)=4 n .
$$

Thus we may define a kind of Gromov-Witten invariants

$$
\Phi_{(\phi, D ; \mathbf{J})}(\alpha, \beta):=\mathrm{EV}^{\phi D} \cdot\left(H \circ\left(f_{M} \times f_{N}\right)\right)
$$

if (51) holds, and zero if (51) does not hold. It is easy to prove that the right side of (52) is independent of the choices of $\hat{J}, g, z_{k}$ and generic representatives. When $\Gamma_{\omega}$ is finitely generated the rational Novikov ring of it is well-defined and thus quantum homology $Q H_{*}(V)$ can be defined as in $\S 4$. In this case we use the idea from [LMP] to define the formal sum

$$
\Psi_{\phi, D}^{\mathbf{J}}(\alpha)=\sum_{B \in \Gamma_{\omega}} \alpha_{B} \otimes e^{B}
$$

for $\alpha \in H_{*}(V, \mathbb{Z})$, where $\alpha_{B} \in H_{*+d+2 c_{1}(B)}(V)$ is determined by

$$
\alpha_{B} \cdot V \beta=\Phi_{(\phi, D+B ; \mathbf{J})}(\alpha, \beta)
$$

for every $\beta \in H_{*}(V, \mathbb{Z})$ and $B \in \Gamma_{\omega}$. Here

$$
d=c_{1}\left(T P_{\phi}^{\text {vert }}\right)(D)
$$

and $D+B$ is understood as in Lemma 2.10 of [Se1], that is, $D+B$ is the only $\Gamma_{\omega}$-equivalence class of sections of $P_{\phi}$ such that

$$
\begin{gathered}
\widetilde{\Omega}_{\phi}(D+B)=\widetilde{\Omega}_{\phi}(D)+\omega(B) \quad \text { and } \\
c_{1}\left(T P_{\phi}^{\text {vert }}\right)(D+B)=c_{1}\left(T P_{\phi}^{\text {vert }}\right)(D)+c_{1}(B) .
\end{gathered}
$$

The following lemma shows that for every $\alpha \in H_{*}(V, \mathbb{Z}), \Psi_{\phi, D}(\alpha)$ is an element of $Q H_{*+d}(V)$.

Lemma 6.5. If $\Gamma_{\omega}$ is finitely generated then for any $\alpha \in H_{*}(V, \mathbb{Z}), \Gamma_{\omega}$ equivalence class $D$ of sections of $P_{\phi}$ and constant $C>0$ there are only finitely many $B \in \Gamma_{\omega}$ such that $\alpha_{B} \neq 0$ and $\omega(B) \leq C$ in (53).

Proof. Since $\Gamma_{\omega}$ is finitely generated the rational Novikov ring of it is a countable set. Moreover, $\alpha_{B} \cdot V \beta=0$ unless $\operatorname{deg}(\alpha)+\operatorname{deg}(\beta)+2 c_{1}\left(T P_{\phi}^{\text {vert }}\right)(D+$ 
$B)=2 n$. Assume that there are a constant $C>0$ and infinitely many $B_{i} \in \Gamma_{\omega}$ such that

$$
\alpha_{B_{i}} \neq 0 \quad \text { and } \quad \omega\left(B_{i}\right) \leq C, i=1,2, \ldots
$$

Then there are infinitely many $\beta_{i} \in H_{*}(V, \mathbb{Z})$ such that

$$
\Phi_{\left(\phi, D+B_{i} ; \mathbf{J}\right)}\left(\alpha, \beta_{i}\right) \neq 0, \quad \operatorname{deg}(\alpha)+\operatorname{deg}\left(\beta_{i}\right)+2 c_{1}\left(T P_{\phi}^{\text {vert }}\right)\left(D+B_{i}\right)=2 n
$$

for all $i=1,2, \ldots$. Recall the definition in (52) we can always find $F_{i} \in$ $\operatorname{Diff}(V \times V)$ such that the image sets of all $F_{i} \circ(f \times h)$ are contained in a fixed compact subset $S$ of $V \times V$. In fact, from the proof of Lemma 4.1 one can find $\mathrm{X}_{i} \in \chi^{r}(V \times V, \mathrm{EV}, f \times h)$ with $\left\|\mathrm{X}_{i}\right\|_{g r} \leq 1$ such that $\mathrm{EV}$ is transverse to all $F_{i} \circ(f \times h)$ with $F_{i}:=\mathcal{F}^{r}\left(\mathrm{X}_{i}\right), i=1,2, \ldots$ But that $\left\|\mathrm{X}_{i}\right\|_{g r} \leq 1$ implies that $\left\|\rho_{r}\left(\mathrm{X}_{i}\right)\right\|_{C^{1}} \leq 2$ for all $i \geq 1$. Thus the image sets of all maps $F_{i} \circ(f \times h)$ are contained in a fixed compact subset of $V \times V$, denoted by $S$. The first formula of (56) shows that there exist $\hat{J}$-holomorphic section $s_{i}$ representing the classes $D+B_{i}$ with $\mathrm{EV}^{\phi\left(D+B_{i}\right)}\left(s_{i}\right) \cap S \neq \emptyset$. In particular, there exists a compact subset $K$ of $P_{\phi}$ such that $s_{i}\left(S^{2}\right) \cap K \neq \emptyset$ for all $i=1,2, \ldots$. Now

$$
0 \leq\left(\widetilde{\Omega}+c \pi^{*} \sigma\right)\left(s_{i}\right)=\widetilde{\Omega}(D)+\omega\left(B_{i}\right)+c \int_{S^{2}} \sigma \leq \widetilde{\Omega}(D)+c \int_{S^{2}} \sigma+C
$$

because $D+B_{i}$ is the equivalence classes of sections of $P_{\phi}$ and $\int_{S^{2}} s^{*}\left(\pi^{*} \sigma\right)=$ $\int_{S^{2}}(\pi \circ s)^{*} \sigma=\int_{S^{2}} \sigma$ for every smooth section $s$ of $P_{\phi}$. This shows that there are infinitely many homology classes in $P_{\phi}$ with nonconstant $\hat{J}$-holomorphic spheres representatives whose image intersects with a fixed compact subset $S$ in $P_{\phi}$. It contradicts to Gromov compactness theorem.

Consequently, (53) defines a $\Lambda_{\omega}$-linear homomorphism $\Psi_{\phi, D}^{\mathbf{J}}$ from $Q H_{*}(M)$ to $Q H_{*+d}(M)$ with $d=2 c_{1}\left(T P_{\phi}^{\text {vert }}\right)(D)$. Moreover, if loops $\phi_{t \in[0,1]}$ and $\chi_{t \in[0,1]}$ are homotopic in $\operatorname{Ham}_{0}^{c}(V, \omega)$ there exists a Hamiltonian fibre bundle isomorphism I ${ }^{\phi \chi}$ having CS property from $P_{\phi}$ to $P_{\chi}$. For a $\mathbf{J} \in \mathcal{J}\left(P_{\phi}, \Omega_{\phi}\right)$ and a $\Gamma_{\omega^{-}}$equivalence class $D$ of sections of $P_{\phi}$ the isomorphism $\mathrm{I}^{\phi \chi}$ determines a $\mathrm{I}_{*}^{\phi \chi}(\mathbf{J})$ and a $\Gamma_{\omega}$-equivalence class $\mathrm{I}_{*}^{\phi \chi}(D)$ of sections of $P_{\chi}$. It is not hard to prove that

$$
\Psi_{\phi, D}^{\mathbf{J}}=\Psi_{\chi, \mathrm{I}_{*}^{\phi \chi}(D)}^{\mathrm{I}_{*}^{\phi \chi}(\mathbf{J})}
$$

As in [Se1], [LMP] we have

$$
\Psi_{\phi, D+B}^{\mathbf{J}}=\Psi_{\phi, D}^{\mathbf{J}} \otimes e^{-B}
$$


for every $B \in \Gamma_{\omega}$ and the $\Gamma_{\omega}$-equivalence classes $D$ of sections of $P_{\phi}$, and the following conclusion.

Lemma 6.6. For the constant loop $\phi_{0}=\{i d\}$ and the $\Gamma_{\omega}$ equivalence class $D_{0}$ of the flat section $s_{0}=S^{2} \times\{p t\}$ of $P_{\phi_{0}}=S^{2} \times V$ the map $\Psi_{\phi_{0}, D_{0}}^{\mathrm{J}}$ is the identity map for any $\mathbf{J} \in \mathcal{J}\left(P_{\phi_{0}}, \Omega_{\phi_{0}}\right)$.

Now if a loop $\chi_{t \in[0,1]}$ is homotopic to $\phi_{0}$ in $\operatorname{Ham}_{0}^{c}(V, \omega)$ then there exists a Hamiltonian fibre bundle isomorphism $\mathrm{I}^{\phi_{0} \chi}$ having CS property from $P_{\phi_{0}}$ to $P_{\chi}$. We call $\Gamma_{\omega^{-}}$equivalence class $I_{*}^{\phi_{0} \chi}\left(D_{0}\right)$ of sections of $P_{\chi}$ as the trivial class. It is independent of choice of the isomorphism $\mathrm{I}^{\phi_{0} \chi}$ having CS property. Thus $\Psi_{\chi, T}^{\mathbf{J}}$ is the identity map for the trivial class $T$ and any $\mathbf{J} \in \mathcal{J}\left(P_{\chi}, \Omega_{\chi}\right)$.

As done in [LMP] the key point of the proof of Theorem 6.4 is to prove the composition rule for maps $\Psi_{\phi, D}$. This needs us to consider the relation between $P_{\phi}, P_{\phi}$ and $P_{\psi * \phi}$. However, unlike the case of [LMP] under which there is the only coupling class $u_{\phi}$ corresponding to $\phi$, in our case we need to replace it by a suitable thing. For two smooth loops $\phi_{t \in[0,1]}$ and $\psi_{t \in[0,1]}$ in $\operatorname{Ham}_{0}^{c}(V, \omega)$ we make the following assumptions: for a fixed sufficiently small $\epsilon>0 \phi_{t}=i d$ for $t \notin[1 / 2+\epsilon, 1-\epsilon]$ and $\psi_{t}=i d$ for $t \notin[\epsilon, 1 / 2-\epsilon]$. Notice that they have been extended to $\mathbb{R} 1$-periodically. Let $H_{\phi}: S^{1} \times V \rightarrow \mathbb{R}$ and $H_{\psi}$ : $S^{1} \times V \rightarrow \mathbb{R}$ be the functions with compact supports and generating loops $\phi_{t \in[0,1]}$ and $\psi_{t \in[0,1]}$ respectively. We can require them to satisfy: $H_{\phi}(t, \cdot)=0$ for $t \notin[1 / 2+\epsilon, 1-\epsilon]$ and $H_{\psi}(t, \cdot)=0$ for $t \notin[\epsilon, 1 / 2-\epsilon]$. Denote by

$$
\begin{aligned}
& T_{\epsilon}:=\left\{[r, t]_{+},[r, t]_{-} \in S^{2} \mid 2 / 3 \leq r \leq 1, t \in[1 / 2+\epsilon, 1-\epsilon]\right\}, \\
& T_{\epsilon}^{*}:=\left\{[r, t]_{+},[r, t]_{-} \in S^{2} \mid 2 / 3 \leq r \leq 1, t \in[\epsilon, 1 / 2-\epsilon]\right\}, \\
& S_{+}^{2}:=\left\{[r, t]_{+},[r, t]_{-} \in S^{2} \mid 0 \leq r \leq 1,0<t<1 / 2\right\}, \\
& S_{-}^{2}:=\left\{[r, t]_{+},[r, t]_{-} \in S^{2} \mid 0 \leq r \leq 1,1 / 2<t<1\right\} .
\end{aligned}
$$

Clearly, $T_{\epsilon}$ and $T_{\epsilon}^{*}$ are proper subsets of the open left hemisphere $S_{+}^{2}$ and open right hemisphere $S_{-}^{2}$ respectively. From the previous construction we may know that

$$
\left.P_{\phi}\right|_{S^{2} \backslash T_{\epsilon}}=\left(S^{2} \backslash T_{\epsilon}\right) \times V \text { and }\left.P_{\psi}\right|_{S^{2} \backslash T_{\epsilon}^{*}}=\left(S^{2} \backslash T_{\epsilon}^{*}\right) \times V .
$$

Thus we may construct the fibre sum $P_{\phi} \sharp P_{\psi}$ as follows: gluing $\left.P_{\phi}\right|_{S^{2} \backslash S_{+}^{2}}$ and $\left.P_{\psi}\right|_{S^{2} \backslash S_{-}^{2}}$ along

$$
\left.\partial P_{\phi}\right|_{S^{2} \backslash S_{+}^{2}}=\left\{[r, t]_{+},[r, t]_{-} \in S^{2} \mid t=0,1 / 2\right\} \times V=\left.\partial P_{\phi}\right|_{S^{2} \backslash S_{-}^{2}}
$$


by the map: $[r, 0, x]_{ \pm}^{\phi} \rightarrow[r, 0, x]_{ \pm}^{\psi}$, and $[r, 1 / 2, x]_{ \pm}^{\phi} \rightarrow[r, 1 / 2, x]_{ \pm}^{\psi}$.

On the other hand it is easy to know that under our assumptions the composite loop $(\phi * \psi)_{t \in[0,1]}=\left(\phi_{t} \circ \psi_{t}\right)_{t \in[0,1]}$ is generated by the Hamiltonian function $H_{\phi * \psi}: S^{1} \times V \rightarrow \mathbb{R}$ given by

$$
H_{\phi * \psi}(t, x)= \begin{cases}H_{\psi}(t, x), & \text { if } 0 \leq t \leq 1 / 2 \\ H_{\phi}(t, x), & \text { if } 1 / 2 \leq t \leq 1\end{cases}
$$

Notice that the Hamiltonian forms $\widetilde{\Omega}_{\phi}$ on $P_{\phi}$ and $\widetilde{\Omega}_{\psi}$ on $P_{\psi}$ constructed as before satisfies:

$$
\widetilde{\Omega}_{\phi}\left|P_{\phi}\right|_{S^{2} \backslash T_{\epsilon}}=p_{2}^{*} \omega, \quad \widetilde{\Omega}_{\psi}\left|P_{\psi}\right|_{S^{2} \backslash T_{\epsilon}^{*}}=p_{2}^{*} \omega .
$$

Hence under the fibre sum operation they define a closed two-form $P_{\phi} \sharp P_{\psi}$, denoted by $\widetilde{\Omega}_{\phi} \sharp \widetilde{\Omega}_{\psi}$. From the above construction it is easily checked that $P_{\phi} \sharp P_{\psi}=P_{\phi * \psi}$ and the closed two-form $\widetilde{\Omega}_{\phi} \sharp \widetilde{\Omega}_{\psi}$ is exactly $\widetilde{\Omega}_{\phi * \psi}$ constructed in the previous way, that is,

$$
\widetilde{\Omega}_{\phi} \sharp \widetilde{\Omega}_{\psi}=\widetilde{\Omega}_{\phi * \psi} .
$$

Now for given sections $s$ of $P_{\phi}$ and $s^{\prime}$ of $P_{\psi}$, by the section homotopy we assume that the restriction of $s$ to $S^{2} \backslash T_{\epsilon / 2}$ and that of $s^{\prime}$ to $S^{2} \backslash T_{\epsilon / 2}^{*}$ have the following versions respectively,

$$
s(z)=\left(z, v_{0}\right), z \in S^{2} \backslash T_{\epsilon / 2}, \quad \text { and } \quad s^{\prime}(z)=\left(z, v_{0}\right), z \in S^{2} \backslash T_{\epsilon / 2}^{*}
$$

for some fixed $v_{0} \in V$. Hence they fit together to give one section of the bundle $P_{\phi} \sharp P_{\psi}$, denoted by

$$
s \sharp s^{\prime} .
$$

Combing (63) with (65) we get

$$
\widetilde{\Omega}_{\phi}(s)+\widetilde{\Omega}_{\psi}\left(s^{\prime}\right)=\widetilde{\Omega}_{\phi} \sharp \widetilde{\Omega}_{\psi}\left(s \sharp s^{\prime}\right)=\widetilde{\Omega}_{\phi * \psi}\left(s \sharp s^{\prime}\right) .
$$

For such chosen sections $s$ and $s^{\prime}$ it follows from (59) that

$$
c_{1}\left(T P_{\phi}^{\mathrm{vert}}\right)(s)+c_{1}\left(T P_{\psi}^{\mathrm{vert}}\right)\left(s^{\prime}\right)=c_{1}\left(T P_{\phi * \psi}^{\mathrm{vert}}\right)\left(s \sharp s^{\prime}\right) .
$$

In fact, since $c_{1}\left(T P_{\phi}^{\text {vert }}\right)(s)=c_{1}\left(s^{*} T P_{\phi}^{\text {vert }}\right)\left(\left[S^{2}\right]\right)$, by the well-known Splitting Principle we only need to consider the case of complex line bundle on $S^{2}$. The latter case may be directly proved with Theorem 2.71 in [McSa2]. 
Notice that (66) and (67) lead to a natural map from

$$
\Gamma_{\omega}\left(P_{\phi}\right) \times \Gamma_{\omega}\left(P_{\psi}\right) \rightarrow \Gamma_{\omega}\left(P_{\phi * \psi}\right),\left(D, D^{\prime}\right) \rightarrow D \sharp D^{\prime},
$$

where $\Gamma_{\omega}\left(P_{\phi}\right), \Gamma_{\omega}\left(P_{\psi}\right)$ and $\Gamma_{\omega}\left(P_{\phi * \psi}\right)$ are the sets of $\Gamma_{\omega}$-equivalence classes of the sections of the bundles $P_{\phi}, P_{\psi}$ and $P_{\phi * \psi}$ respectively. Similarly, since $\mathcal{J}\left(P_{\phi}, \Omega_{\phi}\right)$ and $\mathcal{J}\left(P_{\psi}, \Omega_{\psi}\right)$ are contractible using (59) we always choose $\mathbf{J} \in \mathcal{J}\left(P_{\phi}, \Omega_{\phi}\right)$ and $\mathbf{J}^{\prime} \in \mathcal{J}\left(P_{\psi}, \Omega_{\psi}\right)$ such that

$$
J_{z}=J=J_{w}^{\prime}, \forall z \in S^{2} \backslash T_{\epsilon / 2} \text { and } \forall w \in S^{2} \backslash T_{\epsilon / 2}^{*}
$$

where $J \in \mathcal{J}(V, \omega)$ such that $(V, \omega, J, g)$ is g.bounded for $g \in \mathcal{G}(V)$. Then $\mathbf{J}$ and $\mathbf{J}^{\prime}$ fit together to give one element in $\mathcal{J}\left(P_{\phi * \psi}, \widetilde{\Omega}_{\phi * \psi}\right)$, denoted by $\mathbf{J} \sharp \mathbf{J}^{\prime}$. What we wish to prove is the following composition rule.

Proposition 6.7. For any $\Gamma_{\omega}$-equivalence classes $D$ of sections of $P_{\phi}$ and $D^{\prime}$ of sections of $P_{\psi}$ it holds that

$$
\Psi_{\psi, D^{\prime}}^{\mathbf{J}^{\prime}} \circ \Psi_{\phi, D}^{\mathbf{J}}=\Psi_{\phi * \psi, D \sharp D^{\prime}}^{\mathbf{J} \sharp \mathbf{J}^{\prime}}
$$

Before giving its proof we make an notation:

Remark 6.8. The above base spaces of $P_{\phi}$ and $P_{\psi}$ are denoted by $S_{L}^{2}$ and $S_{R}^{2}$ respectively. Moreover, when constructing the fibre sum $P_{\phi} \sharp P_{\psi}$ we will glue $\left.P_{\phi}\right|_{S^{2} \backslash S_{L \varepsilon}^{2}}$ and $\left.P_{\psi}\right|_{S^{2} \backslash S_{R \varepsilon}^{2}}$ along boundaries $\left.\partial P_{\phi}\right|_{S^{2} \backslash S_{L \varepsilon}^{2}}$ and $\left.\partial P_{\psi}\right|_{S^{2} \backslash S_{R \varepsilon}^{2}}$ by the map

$$
\left[\frac{\cos \varepsilon}{\cos (t+\varepsilon)}, t, x\right]^{\phi} \mapsto\left[\frac{\cos \varepsilon}{\cos (t-\pi)}, t-\pi, x\right]^{\psi}
$$

where

$$
\begin{aligned}
& S_{L \varepsilon}^{2}:=\left\{[r, t]_{+},[r, t]_{-} \in S^{2} \mid \frac{\cos \varepsilon}{\cos t}<r \leq 1,-\varepsilon<t<\varepsilon\right\}, \\
& S_{R \varepsilon}^{2}:=\left\{[r, t]_{+},[r, t]_{-} \in S^{2} \mid \frac{\cos \varepsilon}{\cos (t+\varepsilon)}<r \leq 1, \pi-\varepsilon<t<\pi+\varepsilon\right\} .
\end{aligned}
$$

We denote the fibre sum by $P_{\phi} \sharp_{\varepsilon} P_{\psi}$. Notice that there exists the canonical fibre bundle isomorphism $\mathbf{I}_{\varepsilon}$ from $P_{\phi} \sharp_{\varepsilon} P_{\psi}$ to $P_{\phi} \sharp P_{\psi}$. Later, when saying $\widetilde{\Omega}_{\phi * \psi}$ on $P_{\phi \sharp_{\varepsilon}} P_{\psi}$ and $c_{1}\left(T P_{\phi * \psi}^{\text {vert }}\right)$ we always mean them to be the pullback of $\widetilde{\Omega}_{\phi * \psi}$ and $c_{1}\left(T P_{\phi * \psi}^{\mathrm{vert}}\right)$ on $P_{\phi * \psi}$ under $\mathbf{I}_{\varepsilon}^{*}$ without special statements. The sum $s \sharp s^{\prime}$ of sections and other related objects will be understood similarly. 
Denote by $d=c_{1}\left(T P_{\phi}^{\text {vert }}\right)(D)$ and $d^{\prime}=c_{1}\left(T P_{\phi}^{\text {vert }}\right)\left(D^{\prime}\right)$. By $(67)$ it holds that

$$
c_{1}\left(T P_{\phi * \psi}^{\text {vert }}\right)\left(D \sharp D^{\prime}\right)=d+d^{\prime} .
$$

Thus both $\Psi_{\psi, D^{\prime}}^{\mathbf{J}^{\prime}} \circ \Psi_{\phi, D}^{\mathbf{J}}$ and $\Psi_{\phi * \psi, D \sharp D^{\prime}}^{\mathbf{J} \sharp \mathbf{J}^{\prime}}$ are the homomorphisms from $Q H_{*}(M)$ to $Q H_{*+d+d^{\prime}}(M)$. For a given $\alpha \in H_{*}(M, \mathbb{Z})$ the straightforward computations shows

$$
\Psi_{\psi, D^{\prime}}^{\mathbf{J}^{\prime}} \circ \Psi_{\phi, D}^{\mathbf{J}}(\alpha)=\sum_{A \in \Gamma_{\omega}}\left(\sum_{B \in \Gamma_{\omega}} \alpha_{B, A-B}\right) \otimes e^{A}
$$

where $\alpha_{B, A-B} \in H_{*}(V)$ is determined by

$$
\alpha_{B, A-B} \cdot V \beta=\Phi_{\left(\psi, D^{\prime}+A-B\right)}\left(\alpha_{B}, \beta\right), \forall \beta \in H_{*}(V),
$$

and $\alpha_{B} \in H_{*}(V)$ by

$$
\alpha_{B} \cdot V \gamma=\Phi_{(\phi, D+B)}(\alpha, \gamma), \forall \gamma \in H_{*}(V)
$$

Notice that we also have

$$
\begin{aligned}
\operatorname{dim} \alpha_{B, A-B} & =\operatorname{dim} \alpha_{B}+2 c_{1}\left(T P_{\psi}^{\mathrm{vert}}\right)\left(D^{\prime}\right)+2 c_{1}(A-B) \\
\operatorname{dim} \alpha_{B} & =\operatorname{dim} \alpha+2 c_{1}\left(T P_{\phi}^{\mathrm{vert}}\right)(D)+2 c_{1}(B) .
\end{aligned}
$$

Moreover, by definition we also have

$$
\Psi_{\phi * \psi, D \sharp D^{\prime}}^{\mathbf{J} \sharp \mathbf{J}^{\prime}}(\alpha)=\sum_{A \in \Gamma_{\omega}} \hat{\alpha}_{A} \otimes e^{A},
$$

where $\hat{\alpha}_{A} \in H_{*}(V)$ is determined by

$$
\begin{gathered}
\hat{\alpha}_{A} \cdot V \gamma=\Phi_{\left(\phi * \psi, D \sharp D^{\prime}+A\right)}(\gamma), \gamma \in H_{*}(V), \\
\operatorname{dim} \hat{\alpha}_{A}=\operatorname{dim} \alpha+2 c_{1}\left(T P_{\phi * \psi}^{\mathrm{vert}}\right)\left(D \sharp D^{\prime}\right)+2 c_{1}(A) .
\end{gathered}
$$

Thus we only need to prove that

$$
\hat{\alpha}_{A}=\sum_{B \in \Gamma_{\omega}} \alpha_{B, A-B}, \forall A \in \Gamma_{\omega} .
$$

To complete the proof of Proposition 6.7 we need several lemmas.

Lemma 6.9. For every fixed $A \in H_{*}(V)$ the sum of right side in (81) is always finite. 
Lemma 6.10. There exist the regular almost complex structures $\hat{J}$ on $P_{\phi}$ and $\hat{J}^{\prime}$ on $P_{\psi}$ such that they agree on gluing domain of $P_{\phi} \sharp_{\varepsilon} P_{\psi}$.

Without special statements we shall fix $\hat{J}$ and $\hat{J}^{\prime}$. The proof of Lemma 6.9 is given after Lemma 6.12 and Lemma 6.10 will be proved at the end of this section.

Following the notations in $\S 4$.

Lemma 6.11. Let $e_{1}: U \rightarrow V$ and $e_{2}: U \rightarrow V$ be two $C^{p}$-smooth pseudocycles, and $\alpha: A \rightarrow V$ and $\beta: B \rightarrow V$ be two $C^{q}$-smooth pseudo-cycles. Assume that

$$
\operatorname{dim} U+\operatorname{dim} A+\operatorname{dim} B \geq 2 \operatorname{dim} V,
$$

then for every sufficiently large integer $r>\min \{p, q\}$ there exists a set $\chi^{r}\left(V, e_{1}, e_{2}, \alpha, \beta\right) \subset \chi^{r}(V)_{0} \times \chi^{r}(V)_{0}$ of the second category such that $e=\left(e_{1}, e_{2}\right)$ is transverse to $\left(\mathcal{F}^{r}(\mathrm{X}) \circ \alpha\right) \times\left(\mathcal{F}^{r}(\mathrm{Y}) \circ \beta\right)$ for all $(\mathrm{X}, \mathrm{Y}) \in$ $\chi^{r}\left(V, e_{1}, e_{2}, \alpha, \beta\right)$. These $\chi^{r}\left(V, e_{1}, e_{2}, \alpha, \beta\right)$ also satisfy:

$$
\chi^{r}\left(V, e_{1}, e_{2}, \alpha, \beta\right) \supseteq \chi^{r+1}\left(V, e_{1}, e_{2}, \alpha, \beta\right) \supseteq \ldots,
$$

which implies that for any $(\mathrm{X}, \mathrm{Y}) \in \chi^{r}\left(V, e_{1}, e_{2}, \alpha, \beta\right)$ and $\left(\mathrm{X}^{\prime}, \mathrm{Y}^{\prime}\right) \in$ $\chi^{s}\left(V, e_{1}, e_{2}, \alpha, \beta\right)$ with $s>r$ it holds that

$$
\left[\left(\mathcal{F}^{r}(\mathrm{X}) \circ \alpha\right) \times\left(\mathcal{F}^{r}(\mathrm{Y}) \circ \beta\right)\right] \cdot e=\left[\left(\mathcal{F}^{s}\left(\mathrm{X}^{\prime}\right) \circ \alpha \times\left(\mathcal{F}^{s}\left(\mathrm{Y}^{\prime}\right)\right] \cdot e\right.\right.
$$

provided that the equality in (83) holds, and one of $e=\left(e_{1}, e_{2}\right)$ and $\alpha \times \beta$ is the strong pseudo-cycle.

The proof of this lemma is similar to that of Lemma 4.1. Replacing (27) one only consider the map

$$
\Xi: U \times A \times B \times \chi^{r}(V)_{0} \times \chi^{r}(V)_{0} \rightarrow V \times V \times V \times V
$$

given by

$$
(u, a, b, \mathrm{X}, \mathrm{Y}) \mapsto\left(\left(e_{1}(u), e_{2}(u)\right),\left(\mathcal{F}^{r}(\mathrm{X})(\alpha(a)), \mathcal{F}^{r}(\mathrm{Y})(\beta(b))\right)\right) .
$$

It is easy to prove that it is transverse to

$$
\Delta_{V \times V}:=\{(u, v, u, v) \mid(u, v) \in V \times V\} .
$$

The standard arguments may finish the proof. 
By (i) of Lemma 4.1 one know that if

$$
\operatorname{dim} U+\operatorname{dim} A \geq \operatorname{dim} V
$$

then for every sufficiently large integer $r>\min \{p, q\}$ there exists a set $\chi^{r}\left(V, e_{1}, \alpha\right) \subset \chi^{r}(V)_{0}$ of the second category such that $e_{1}$ is transverse to $\mathcal{F}^{r}(\mathrm{X}) \circ \alpha$ for all $\mathrm{X} \in \chi^{r}\left(V, e_{1}, \alpha\right)$. From Claim A.1.11 of [LeO] the space

$$
\chi_{1}^{r}\left(V, e_{1} \times e_{2}, \alpha, \beta\right)
$$

consisting of all $\mathrm{X} \in \chi^{r}(V)_{0}$ for which the intersection

$$
\chi^{r}\left(V, e_{1}, e_{2}, \alpha, \beta\right) \cap\left[\{\mathrm{X}\} \times \chi^{\mathrm{r}}(\mathrm{V})_{0}\right]
$$

is a countable intersection of open dense subset in $\{\mathrm{X}\} \times \chi^{\mathrm{r}}(\mathrm{V})_{0}$ must be a countable intersection of open dense subsets in $\chi^{r}(V)_{0}$. Thus the intersection

$$
\chi_{1}^{r}\left(V, e_{1} \times e_{2}, \alpha, \beta\right) \cap \chi^{r}\left(V, e_{1}, \alpha\right)
$$

is also a countable intersection of open dense subsets in $\chi^{r}(V)_{0}$. For every $\mathrm{X}$ in this intersection there must be a $\mathrm{Y} \in \chi^{r}(V)_{0}$ such that $(\mathrm{X}, \mathrm{Y}) \in$ $\chi^{r}\left(V, e_{1} \times e_{2}, \alpha, \beta\right)$. Thus this pair $(\mathrm{X}, \mathrm{Y})$ satisfies:

(i) $e=\left(e_{1}, e_{2}\right)$ is transverse to $\left(\mathcal{F}^{r}(\mathrm{X}) \circ \alpha\right) \times\left(\mathcal{F}^{r}(\mathrm{Y}) \circ \beta\right)$,

(ii) $e_{1}$ is transverse to $\mathcal{F}^{r}(\mathrm{X}) \circ \alpha$

under the assumptions (83), (84).

Lemma 6.12. Let $e_{i}: U \rightarrow V, i=1,2$ and $\alpha: A \rightarrow V$ and $\beta: B \rightarrow V$ be all $C^{r}$-smooth pseudo-cycles. Assume that $e=\left(e_{1}, e_{2}\right)$ is transverse to $\alpha \times \beta, e_{1}$ is transverse to $\alpha$ and (83), (84) also hold. Then

$$
\Delta(U \times A):=\left\{(u, a) \mid e_{1}(u)=\alpha(a)\right\}
$$

is a $C^{r}$-smooth manifold of dimension $\operatorname{dim} U+\operatorname{dim} A-\operatorname{dim} V$, and

$$
\hat{e}_{2}: \Delta(U \times A) \rightarrow V,(u, a) \mapsto e_{2}(u)
$$

is also $C^{r}$-smooth pseudo-cycle which is transverse to $\beta$. Moreover, if $\alpha$ and $\beta$ are strong pseudo-cycle then it holds that

$$
e \cdot(\alpha \times \beta)=\hat{e}_{2} \cdot \beta .
$$


Proof. Let $\hat{e}_{2}(u, a)=\beta(b)$. We wish to prove

$$
D \hat{e}_{2}(u, a)\left(T_{(u, a)} \Delta(U \times A)\right)+D \beta(b)\left(T_{b} B\right)=T_{\beta(b)} V .
$$

Notice that

$$
T_{(u, a)} \Delta(U \times A)=\left\{(\vec{u}, \vec{a}) \in T_{u} U \times T_{a} A \mid D e_{1}(u)(\vec{u})=D \alpha(a)(\vec{a})\right\} .
$$

It suffice to prove that for any $\xi \in T_{\beta(b)} V$ there exist $\vec{u} \in T_{u} U, \vec{a} \in T_{a} A$ and $\vec{b} \in T_{b} B$ such that

$$
D e_{1}(u)(\vec{u})=D \alpha(a)(\vec{a}) \quad \text { and } \quad D e_{2}(u)(\vec{u})+D \beta(b)(\vec{b})=\xi .
$$

But $e$ is transverse to $\alpha \times \beta$. Therefore, there exist $(\vec{u}, \vec{a}, \vec{b}) \in T_{u} U \times T_{a} A \times T_{b} V$ such that

$$
D e(u)(\vec{u})+D(\alpha \times \beta)(a, b)(-\vec{a}, \vec{b})=(0, \xi) .
$$

Clearly, this is equivalent to (90). By similar arguments for the boundary parts we can prove that $\hat{e}_{2}$ is a $C^{r}$-smooth pseudo-cycle which is transverse to $\beta$.

Notice that $\hat{e}_{2}$ is also a strong pseudo-cycle if $\alpha$ is. Now $e \cdot(\alpha \times \beta)$ and $\hat{e}_{2} \cdot \beta$ are well-defined. To prove them being equal we notice that

$$
\begin{aligned}
\hat{e}_{2} \cdot \beta & =\sum_{\substack{e_{1}(u)=\alpha(a) \\
e_{2}(u)=\beta(b)}} \operatorname{sign}((u, a), b) \\
& =\sum_{\substack{e_{1}(u)=\alpha(a) \\
e_{2}(u)=\beta(b)}} \operatorname{sign}(u, a, b), \\
e \cdot(\alpha \times \beta) & =\sum_{\substack{e(u)=(\alpha \times \beta)(a, b) \\
e^{\prime}}} \operatorname{sign}(u,(a, b)) \\
& =\sum_{\substack{e_{1}(u)=\alpha(a) \\
e_{2}(u)=\beta(b)}} \operatorname{sign}(u, a, b) .
\end{aligned}
$$

Here some details on the orientation are omitted. It is not very difficult to give them. At least, for the mod 2 intersection number the above arguments is completed. The lemma is proved.

Remark 6.13. Using Lemma 6.12 we may give a pseudo-cycle expression of $\alpha_{B}$ in (75) as follows: Firstly, by Lemma $6.11 H \in \operatorname{Diff}(V \times V)$ in (52) 
may be chosen as the form $H=\left(h_{1}, h_{2}\right)$ with $h_{i} \in \operatorname{Diff}(V), i=1,2$. Thus (75) becomes

$$
\alpha_{B} \cdot V \gamma=\mathrm{EV}^{\phi(D+B)} \cdot\left(h_{1} \circ f_{M} \times h_{2} \circ f_{N}\right),
$$

where $f_{M}: M \rightarrow V$ and $f_{N}: N \rightarrow V$ are the strong pseudo-cycle representatives of $\alpha$ and $\gamma$ respectively, $h_{i} \in \operatorname{Diff}(V), i=1,2$, and

$$
\begin{array}{r}
\mathrm{EV}^{\phi(D+B)}=\left(\mathrm{EV}_{1}^{\phi(D+B)}, \mathrm{EV}_{2}^{\phi(D+B)}\right): \mathcal{S}\left(P_{\phi}, \Omega_{\phi}, j, \hat{J}, D+B\right) \rightarrow V \times V, \\
s \mapsto\left(F_{1}^{\phi}\left(s\left(z_{1}^{\phi}\right)\right), F_{2}^{\phi}\left(s\left(z_{2}^{\phi}\right)\right)\right)
\end{array}
$$

is the pseudo-cycle determined by the evaluation map. By lemma 6.12 the right side of (91) is equal to

$$
\widehat{\mathrm{EV}}_{2}^{\phi(D+B)} \cdot\left(h_{2} \circ f_{N}\right),
$$

where the pseudo-cycle

$$
\widehat{\mathrm{EV}}_{2}^{\phi(D+B)}: \Delta\left(\mathcal{S}\left(P_{\phi}, \Omega_{\phi}, j, \hat{J}, D+B\right), M\right) \rightarrow V
$$

given by

$$
(s, a) \mapsto F_{2}^{\phi}\left(s\left(z_{2}^{\phi}\right)\right) .
$$

By definition

$$
\begin{aligned}
& \Delta\left(\mathcal{S}\left(P_{\phi}, \Omega_{\phi}, j, \hat{J}, D+B\right), M\right) \\
& =\left\{(s, a) \in \mathcal{S}\left(P_{\phi}, \Omega_{\phi}, j, \hat{J}, D+B\right) \times M \mid F_{1}^{\phi}\left(s\left(z_{1}^{\phi}\right)\right)=h_{1} \circ f_{M}(a)\right\} .
\end{aligned}
$$

Thus (93) may be considered as a pseudo-cycle representative of $\alpha_{B}$.

Proof of Lemma 6.9. Assume that there exists $A \in \Gamma_{\omega}$ such that $\alpha_{B, A-B} \neq 0$ for infinitely many $B \in \Gamma_{\omega}$. Denote them by $B_{1}, B_{2}, \ldots$ By Remark 6.13 one gets infinitely many pseudo-cycles

$$
\widehat{\mathrm{EV}}_{2}^{\phi\left(D+B_{i}\right)}: \Delta\left(\mathcal{S}\left(P_{\phi}, \Omega_{\phi}, j, \hat{J}, D+B_{i}\right), M\right) \rightarrow V
$$

$$
\begin{aligned}
& \Delta\left(\mathcal{S}\left(P_{\phi}, \Omega_{\phi}, j, \hat{J}, D+B_{i}\right), M\right) \\
& =\left\{(s, a) \in \mathcal{S}\left(P_{\phi}, \Omega_{\phi}, j, \hat{J}, D+B_{i}\right) \times M \mid F_{1}^{\phi}\left(s\left(z_{1}^{\phi}\right)\right)=h_{1}^{(i)} \circ f_{M}(a)\right\}
\end{aligned}
$$


for some $h_{1}^{(i)} \in \operatorname{Diff}(V)$. From Lemma 6.11 and the arguments under it one can assume all $h_{1}^{(i)}$ to be the same $h_{1}$. But the image of $h_{1} \circ f_{M}$ is contained in a compact subset of $V$. From the results in $\S 2$ it follows that the image sets of all sections $s$ which are such that

$$
(\{s\} \times M) \bigcap\left(\bigcup \Delta\left(\mathcal{S}\left(P_{\phi}, \Omega_{\phi}, j, \hat{J}, D+B_{i}\right), M\right)\right) \neq \emptyset,
$$

are contained in a compact subset of $P_{\phi}$. Thus the image sets of all such pseudo-cycles representatives of $\alpha_{B_{i}}$ given by Remark 6.13 are contained in a compact subset $K(\phi)$ of $V$. By the assumption at the beginning

$$
\alpha_{B_{i}, A-B_{i} \cdot V} \beta_{i} \neq 0, \text { for some } \beta_{i} \in H_{*}(V)
$$

Now from (74) it follows that there exist sections

$$
s_{i}^{\prime} \in \mathcal{S}\left(P_{\psi}, \Omega_{\psi}, j, \hat{J}^{\prime}, D^{\prime}+A-B_{i}\right)
$$

such that $\mathrm{EV}_{1}^{\psi\left(D^{\prime}+A-B_{i}\right)}\left(s_{i}^{\prime}\right)=F_{1}^{\psi}\left(s_{i}^{\prime}\left(z_{1}^{\psi}\right)\right)$ are contained in the compact subset $K(\phi)$. Hence the image sets of all sections $s_{i}^{\prime}$ are contained in a compact subset $\mathrm{S}(\psi)$ of $P_{\psi}$. Because all $s_{i}^{\prime}$ are $\left(j, \hat{J}^{\prime}\right)$-holomorphic it holds that

$$
\left(\widetilde{\Omega}_{\psi}+c_{0} \sigma\right)\left(s_{i}^{\prime}\right) \geq 0, i=1,2, \ldots,
$$

which implies

$$
\omega\left(B_{i}\right)=\left(\widetilde{\Omega}_{\psi}+c_{0} \sigma\right)\left(B_{i}\right) \leq\left(\widetilde{\Omega}_{\psi}+c_{0} \sigma\right)\left(D^{\prime}+A\right), i=1,2, \ldots
$$

Hence

$$
\left(\widetilde{\Omega}_{\phi}+c_{0} \sigma\right)\left(D+B_{i}\right) \leq\left(\widetilde{\Omega}_{\phi}+c_{0} \sigma\right)(D)+\left(\widetilde{\Omega}_{\psi}+c_{0} \sigma\right)\left(D^{\prime}+A\right), i=1,2, \ldots
$$

Take $\left(s_{i}, a_{i}\right) \in \Delta\left(\mathcal{S}\left(P_{\phi}, \Omega_{\phi}, j, \hat{J}, D+B_{i}\right), M\right)$ one gets infinitely many $(j, \hat{J})$ holomorphic sections $\left\{s_{i}\right\}$ which represent infinitely many different classes and whose image sets are contained in a fixed compact subset of a g.bounded symplectic manifold $\left(P_{\phi}, \widetilde{\Omega}_{\phi}+c_{0} \sigma, \hat{J}, g\right)$. With the same reason as in Lemma 6.5 (98) leads to a contradiction.

Now we have known that the sum on the right side of (81) is actually finite sum. To prove (81) holding let us check their pseudo-cycle representatives given by Remark 6.13. Using the pseudo-cycles representative of 
$\alpha_{B}$ given by (93) one may get a pseudo-cycle representative of $\alpha_{B, A-B}$ as follows:

$$
\begin{aligned}
\widehat{\mathrm{EV}}_{2}^{\psi\left(D^{\prime}+A-B\right)}: \Delta\left(\mathcal{S}\left(P_{\psi}, \Omega_{\psi}, j, \hat{J}^{\prime}, D^{\prime}+A-B\right),\right. & \\
\left.\Delta\left(\mathcal{S}\left(P_{\phi}, \Omega_{\phi}, j, \hat{J}, D+B\right), M\right)\right) & \rightarrow V
\end{aligned}
$$

given by

$$
\left(s^{\prime},(s, a)\right) \mapsto F_{2}^{\psi}\left(s^{\prime}\left(z_{2}^{\psi}\right)\right) .
$$

By definition it is easy to check that the set in (99) consists of all triples $\left(s^{\prime}, s, a\right)$ satisfying the conditions

$$
\left.\begin{array}{c}
s^{\prime} \in \mathcal{S}\left(P_{\psi}, \Omega_{\psi}, j, \hat{J}^{\prime}, D^{\prime}+A-B\right) \\
s \in \mathcal{S}\left(P_{\phi}, \Omega_{\phi}, j, \hat{J}, D+B\right) \\
a \in M \\
F_{2}^{\phi}\left(s\left(z_{2}^{\phi}\right)\right)=h_{1}^{\phi(D+B)} \circ f_{M}(a) \\
F_{1}^{\psi}\left(s^{\prime}\left(z_{1}^{\psi}\right)\right)=h_{1}^{\psi\left(D^{\prime}+A-B\right)} \circ F_{2}^{\phi}\left(s\left(z_{2}^{\phi}\right)\right)
\end{array}\right\}
$$

for some $h_{1}^{\phi(D+B)}$ and $h_{1}^{\psi\left(D^{\prime}+A-B\right)}$ in $\operatorname{Diff}(V)$. Moreover, from Lemma 6.12 it is easily computed that the dimension of manifold in (99) is

$$
\operatorname{dim} \alpha+2 c_{1}(A)+2 c_{1}\left(T P_{\phi * \psi}^{\mathrm{vert}}\right)\left(D \sharp D^{\prime}\right) .
$$

On the other hand $\hat{\alpha}_{A}$ has the pseudo-cycle representative:

$$
\widehat{\mathrm{EV}}_{2}^{\phi * \psi\left(D \sharp D^{\prime}+A\right)}: \Delta\left(\mathcal{S}\left(P_{\phi * \psi}, \Omega_{\phi * \psi}, j, \hat{J} \sharp \hat{J}^{\prime}, D \sharp D^{\prime}+A\right), M\right) \rightarrow V
$$

given by

$$
(\sigma, a) \mapsto F_{2}^{\phi * \psi}\left(\sigma\left(z_{2}^{\phi * \psi}\right)\right)
$$

By definition

$$
\Delta\left(\mathcal{S}\left(P_{\phi * \psi}, \Omega_{\phi * \psi}, j, \hat{J} \sharp \hat{J}^{\prime}, D \sharp D^{\prime}+A\right), M\right)
$$

consists of all pairs $(\sigma, a)$ satisfying

$$
\left.\begin{array}{c}
\sigma \in \mathcal{S}\left(P_{\phi * \psi}, \Omega_{\phi * \psi}, j, \hat{J} \sharp \hat{J}^{\prime}, D \sharp D^{\prime}+A\right) \\
a \in M \\
F_{1}^{\phi * \psi}\left(\sigma\left(z_{1}^{\phi * \psi}\right)\right)=h_{1}^{\phi * \psi\left(D \sharp D^{\prime}+A\right)} \circ f_{M}(a)
\end{array}\right\}
$$


for some $h_{1}^{\phi * \psi\left(D \sharp D^{\prime}+A\right)}$ in $\operatorname{Diff}(M)$. Here it should be noted that the choices of $h_{1}^{\phi * \psi\left(D \sharp D^{\prime}+A\right)}$ in (106) and $h_{1}^{\phi(D+B)}$ and $h_{1}^{\psi\left(D^{\prime}+A-B\right)}$ in (101) have the "bigger" freedom. But the choices of $h_{1}^{\psi\left(D^{\prime}+A-B\right)}$ are under the case that $h_{1}^{\phi(D+B)}$ is chosen. Another important point is the maps in (99) and (103) to have precompact image sets in $V$. Thus they are all strong pseudo-cycles in the sense of $\S 4$.

Having the above preparation we may prove (81) and thus finish the proof of Proposition 6.7. We only need to prove

$$
P D\left(\hat{\alpha}_{A}\right)=\sum_{B \in \Gamma_{\omega}} P D\left(\alpha_{B, A-B}\right), \forall A \in \Gamma_{\omega} .
$$

That is, their Poincarè dualities in $H_{c}^{*}(V)$ are same. But (107) is equivalent to

$$
\left\langle P D\left(\hat{\alpha}_{A}\right), \gamma\right\rangle=\sum_{B \in \Gamma_{\omega}}\left\langle P D\left(\alpha_{B, A-B}\right), \gamma\right\rangle, \forall \gamma \in H_{*}(V)
$$

Therefore, one only need to prove that for every $\gamma \in H_{*}(V)$ with

$$
\operatorname{dim} \gamma=\operatorname{dim} \alpha+2 c_{1}(A)+2 c_{1}\left(T P_{\phi * \psi}^{\mathrm{vert}}\right)\left(D \sharp D^{\prime}\right)
$$

we may choose a pseudo-cycle representative of it $\Upsilon: T \rightarrow V$ such that it is transverse to the map in (104) and all maps in (99) and

$$
\Upsilon \cdot \widehat{\mathrm{EV}}_{2}^{\phi * \psi\left(D \sharp D^{\prime}+A\right)}=\sum_{B \in \Gamma_{\omega}} \Upsilon \cdot \widehat{\mathrm{EV}}_{2}^{\psi\left(D^{\prime}+A-B\right)} .
$$

By definitions the left side of (109) is equal to the sum

$$
\sum \operatorname{sign}(r, \sigma, a)
$$

when $(r, \sigma, a)$ takes over the set

$$
\begin{array}{r}
\left\{(r,(\sigma, a)) \in T \times \Delta\left(\mathcal{S}\left(P_{\phi * \psi}, \Omega_{\phi * \psi}, j, \hat{J} \sharp \hat{J}^{\prime}, D \sharp D^{\prime}+A\right), M\right) \mid\right. \\
\left.\Upsilon(r)=F_{2}^{\phi * \psi}\left(\sigma\left(z_{2}^{\phi * \psi}\right)\right)\right\} .
\end{array}
$$

The right side of (109) is equal to the sum

$$
\sum \operatorname{sign}\left(r, s^{\prime}, s, a\right)
$$


where $\left(r,\left(s^{\prime},(s, a)\right)\right)$ takes over the set

$$
\Lambda\left(T, M, P_{\psi}, P_{\phi}, \hat{J}, \hat{J}^{\prime}, A, D, D^{\prime}\right)
$$

consisting of all

$$
\begin{aligned}
&\left(r,\left(s^{\prime},(s, a)\right)\right) \in T \times \\
& \bigcup_{B \in \Gamma_{\omega}} \Delta\left(\mathcal{S}\left(P_{\psi}, \Omega_{\psi}, j, \hat{J}^{\prime}, D^{\prime}+A-B\right), \Delta\left(\mathcal{S}\left(P_{\phi}, \Omega_{\phi}, j, \hat{J}, D+B\right), M\right)\right)
\end{aligned}
$$

such that $\Upsilon(r)=F_{2}^{\psi}\left(s^{\prime}\left(z_{2}^{\psi}\right)\right)$. Notice that two sets in (111) and (113) are finite.

By Remark 6.8 we here may choose

$$
\begin{aligned}
& \left.z_{1}^{\phi}=\left[\frac{5}{6}, \frac{1}{2}\right]_{+}=\left[\frac{5}{6},-\frac{1}{2}\right]_{-}, \quad z_{2}^{\phi}=\left[\frac{5}{6}, 0\right]_{+}=\left[\frac{5}{6}, 0\right]_{-}\right\} \\
& \left.z_{1}^{\psi}=\left[\frac{5}{6}, \frac{1}{2}\right]_{+}=\left[\frac{5}{6},-\frac{1}{2}\right]_{-}, \quad z_{2}^{\psi}=\left[\frac{5}{6}, 0\right]_{+}=\left[\frac{5}{6}, 0\right]_{-}\right\} \text {. }
\end{aligned}
$$

Since the bundle $P_{\psi}$ and $P_{\psi}$ are trivial near $z_{2}^{\phi}$ and $z_{1}^{\psi}$ respectively, one can use the gluing techniques developed in [RT1], [McSa1] to prove that there exists an orientation-preserving bijection between the set in (111) and one in (113). This can lead to (109). Hence the proof of Proposition 6.7 is completed under the assumption that Lemma 6.10 holds.

Proof of Lemma 6.10. Recall the technique used in $\S 2$ and $\S 4$. We only need to prove the following fact:

Fact 6.14. For a Riemannian vector bundle $\pi: E \rightarrow W$, denoted $C_{b}^{0}(E)$ by the Banach space of all bounded continuous sections of $\pi$. A norm of a section $s \in C_{b}^{0}(E)$ is given by

$$
\|s\|:=\sup _{x \in W}\|s(x)\|_{g}
$$

where $g$ is a given Riemannian metric on $E$. Let $W_{0}$ be an open submanifold of $W$. Then for every open dense subset $\mathcal{A}$ in $C_{b}^{0}(E)$ the restriction $\mathcal{A} \mid W_{0}:=$ $\left\{\left.s\right|_{W_{0}} \mid s \in \mathcal{A}\right\}$ is also an open dense subset in $C_{b}^{0}\left(E \mid W_{0}\right)$.

In fact, if there exists an open ball $B\left(s_{0}, \delta\right) \subseteq C_{b}^{0}\left(E \mid W_{0}\right) \backslash \mathcal{A} \mid W_{0}$ then one can find a section $s \in C_{b}^{0}(E)$ such that

$$
\left\|\left.s\right|_{W_{0}}-s_{0}\right\|<\frac{1}{5} \delta .
$$


For this section $s$ there exists a section $s^{\prime} \in \mathcal{A}$ such that

$$
\left\|s-s^{\prime}\right\|<\frac{1}{5} \delta
$$

Specially, this shows that $\left\|\left.s\right|_{W_{0}}-\left.s^{\prime}\right|_{W_{0}}\right\|<\frac{1}{5} \delta$. Thus $\left.s^{\prime}\right|_{W_{0}} \notin \mathcal{A} \mid W_{0}$, which leads to a contradiction.

Now as in [LMP] it follows from Lemma 6.6 and Proposition 6.7 that every $\Psi_{\phi, D}^{\mathbf{J}}$ is an isomorphism which leads to Theorem 6.4.

Remark 6.15. The conclusion of Corollary 6.3 can be actually strengthened to general case, that is, $\operatorname{Diff}(M, \partial M)$ is replaced by $\operatorname{Diff}(M)$. We will outline these as follows. Let $[0,1] \rightarrow \phi_{t}$ be a smooth loop in $\operatorname{Symp}(M, \omega)$, and $(\widetilde{M}, \widetilde{\omega})$ a noncompact symplectic manifold associated to $(M, \omega)$ as in $\S 5$. Here we need to write it in detail. Since $\partial M$ is a hypersurface of contact type, for a contact form $\alpha$ on $\partial M$ with $d \alpha=\left.\omega\right|_{\partial M}$ the standard arguments shows that there exists a $\varepsilon \in(0,1)$ and an embedding $\varphi: \partial M \times[\varepsilon, 1] \rightarrow M$ of codimension zero such that

$$
\varphi(m, 1)=m \quad \text { and } \quad \varphi^{*} \omega=d \Theta \text { on } \partial M \times[\varepsilon, 1]
$$

where $\Theta$ is a one-form on $\partial M \times[\varepsilon,+\infty)$ with $\Theta(m, z)=z \alpha(m)$ at a point $(m, z)$. Then $(\widetilde{M}, \widetilde{\omega})$ can be obtained by gluing $(M, \omega)$ and $(\partial M \times[\varepsilon,+\infty), d \Theta)$ with $\varphi$. That is, $(m, z) \in \partial M \times[\varepsilon, 1]$ and $\varphi(m, z) \in M$ are identified. Notice that $\phi_{t}(\partial M)=\partial M$, one can always find a $\epsilon \in(\varepsilon, 1)$ such that

$$
\bigcup_{t \in[0,1]} \phi_{t} \circ \varphi(\partial M \times(\epsilon, 1])
$$

is contained in $\operatorname{Im}(\varphi)$. Thus every

$$
\varphi^{-1} \circ \phi_{t} \circ \varphi: \partial M \times(\epsilon, 1] \rightarrow \partial M \times(\varepsilon, 1]
$$

is an embedding of codimension zero, and it also holds that

$$
\varphi^{-1} \circ \phi_{t} \circ \varphi(m, 1)=\left(\Phi_{t}(m), 1\right), \quad \forall m \in \partial M
$$

where $\Phi_{t}: \partial M \rightarrow \partial M$ is a smooth family of diffeomorphisms. Since $\left(\varphi^{-1} \circ\right.$ $\left.\phi_{t} \circ \varphi\right)^{*} d \Theta=d \Theta$ it must holds that

$$
\Phi_{t}^{*} \alpha=\alpha
$$


Define

$$
\widetilde{\phi}_{t}: \widetilde{M} \rightarrow \widetilde{M}, q \mapsto \begin{cases}\phi_{t}(q) & \text { on } q \in M \\ \left(\Phi_{t}(m), z\right) & \text { on } q=(m, z) \in \partial M \times[1,+\infty)\end{cases}
$$

It is easily checked that $t \mapsto \widetilde{\phi}_{t}$ is a smooth loop in $\operatorname{Symp}(\widetilde{M}, \widetilde{\omega})$. Moreover, if $\left\{\phi_{t}\right\}_{t \in[0,1]}$ is generated by a smooth function $H: M \times \mathbb{R} / \mathbb{Z} \rightarrow \mathbb{R}$ then $\left\{\widetilde{\phi}_{t}\right\}$ is generated by the smooth function

$$
\widetilde{H}: \widetilde{M} \times \mathbb{R} / \mathbb{Z} \rightarrow \mathbb{R}, \quad(q, t) \mapsto \begin{cases}H(m, t) & \text { if }(q, t)=(m, t) \in M \times \mathbb{R} / \mathbb{Z} \\ H(m, t) & \text { if }(q, t)=((m, z), t) \in \\ & (\partial M \times[1,+\infty)) \times \mathbb{R} / \mathbb{Z} .\end{cases}
$$

Now one may construct a Hamiltonian fibre bundle $P_{\widetilde{\phi}}$ over $S^{2}$ with fibre $(\widetilde{M}, \widetilde{\omega})$. Furthermore, replacing $H$ with $\widetilde{H}$ in the previous construction we may get a Hamiltonian 2 -form $\widetilde{\Omega}_{\widetilde{\phi}}$ on $P_{\widetilde{\phi}}$. An important point is that $\left(P_{\widetilde{\phi}}, \widetilde{\Omega}_{\widetilde{\phi}}, \hat{J}, G\right)$ is also g. bounded for some $\hat{J} \in \widehat{\mathcal{J}}(j, \mathbf{J})$ and some complete Riemannian metric $G$. Suitably modifying the above arguments one may obtain the following corresponding results to Theorem 6.4 and Theorem 6.1 .

Proposition 6.16. For a loop $\phi_{t \in[0,1]}$ in $\operatorname{Ham}(M, \omega)$ and the extension loop $\widetilde{\phi}_{t \in[0,1]}$ in $\operatorname{Ham}(\widetilde{M}, \widetilde{\omega})$ as above, the homomorphism $i: H_{*}(\widetilde{M}, \mathbb{Q}) \rightarrow$ $H_{*}\left(P_{\widetilde{\phi}}, \mathbb{Q}\right)$ is injective. Consequently, the endomorphism $\partial_{\widetilde{\phi}}: H_{*}(\widetilde{M}, \mathbb{Q}) \rightarrow$ $H_{*+1}(\widetilde{M}, \mathbb{Q})$ vanishes. Especially, the endomorphism $\partial_{\phi}: H_{*}(M, \mathbb{Q}) \rightarrow$ $H_{*+1}(M, \mathbb{Q})$ vanishes.

Using this result and the flux homomorphism theorem given in Appendix which is the version of Theorem 10.12 in [McSa2] on the compact symplectic manifold with contact type boundary we get the following strengthened version of Corollary 6.3.

Corollary 6.17. For $\phi \in \pi_{1}(\operatorname{Diff}(M), i d)$ and any two $\omega_{1}$ and $\omega_{2}$ in $\operatorname{Cont}(M)$ it holds that $\phi \in \operatorname{Im}\left(H_{\omega_{1}}\right) \cap \operatorname{Im}\left(S_{\omega_{2}}\right)$ if and only if $\phi \in \operatorname{Im}\left(H_{\omega_{2}}\right) \cap$ $\operatorname{Im}\left(S_{\omega_{1}}\right)$.

Finally, we point out that using results in $\S 5$ one can also generalize Theorem 5.A in [LMP] to the present case. 
Remark 6.18. After this paper had been finished I saw D. McDuff's beautiful paper [Mc2]. It is very possible to use our method to generalize her some results. Moreover, from proof of Theorem 6.4 it easily follows that Theorem 6.4 still holds if the loop $\phi$ belongs to $\operatorname{Ham}(V, \omega)$ rather than $\operatorname{Ham}^{c}(V, \omega)$, but we must require that Hamiltonian function $H_{\phi}: S^{1} \times V \rightarrow \mathbb{R}$ generating $\phi$ satisfies some conditions( for example, a possible choice is one that for some g.bounded Riemannian metric $g$ on $V$ it holds that $\left.\operatorname{Sup}\|d H(t, x)\|_{g}<+\infty\right)$. These will be given in other place.

\section{Appendix.}

Suitably modifying the proof of Theorem 10.12 in [McSa2] one may get the following theorem. For convenience of the readers we shall give its proof.

Theorem A. Let $(M, \omega)$ be a compact symplectic manifold with contact type boundary. Then a smooth path

$$
[0,1] \rightarrow \operatorname{Symp}_{0}(M, \omega): t \mapsto \phi_{t}
$$

from $\phi_{0}=i d$ may be isotopic with fixed endpoints to a Hamiltonian path in $\operatorname{Ham}(M, \omega)$ if and only if $\operatorname{Flux}\left(\left\{\phi_{t}\right\}\right)=0$.

Proof. Firstly, notice that the flux homomorphism is still well-defined on $\widetilde{\operatorname{Symp}}_{0}(M, \omega)$ or even on $\widetilde{\operatorname{Symp}}_{0}(\widetilde{M}, \widetilde{\omega})$ and is indeed a homomorphism because there exists a natural homotopy equivalence between $M$ and $\widetilde{M}$.

Next, we only need to prove the "only if" part. Let $\phi_{t \in[0,1]}$ be a smooth path from $\phi_{0}=i d$ in $\operatorname{Symp}_{0}(M, \omega)$ with $\operatorname{Flux}\left(\left\{\phi_{t}\right\}\right)=0$. As in Remark 6.15 it is extended into a path from $i d$ in $\operatorname{Symp}_{0}(\widetilde{M}, \widetilde{\omega})$, denoted by $\widetilde{\phi}_{t \in[0,1]}$. It has the version as in (117), (118). Denote by

$$
\text { (A.1) } X_{t}=\left(\frac{d}{d t} \phi_{t}\right) \circ \phi_{t}^{-1}, \quad \widetilde{X}_{t}=\left(\frac{d}{d t} \widetilde{\phi}_{t}\right) \circ \widetilde{\phi}_{t}^{-1} \quad \text { and } \quad \mathcal{X}_{t}=\left(\frac{d}{d t} \Phi_{t}\right) \circ \Phi_{t}^{-1}
$$

then

$$
\widetilde{X}_{t}(q)= \begin{cases}X_{t}(m) & \text { if } q=m \in M \\ \left(\mathcal{X}_{t}(m), 0\right) & \text { if } q=(m, z) \in \partial M \times[1,+\infty) .\end{cases}
$$

Thus

$$
i_{\widetilde{X}_{t}} \widetilde{\omega}(q)= \begin{cases}i_{X_{t}} \omega(m) & \text { if } q=m \in M ; \\ -\alpha\left(\mathcal{X}_{t}\right)(m) d z-z d\left(\alpha\left(\mathcal{X}_{t}\right)(m)\right. & \text { if } q=(m, z) \in \partial M \times[1,+\infty) .\end{cases}
$$


Moreover, it always holds that $\operatorname{Flux}\left(\left\{\phi_{t}\right\}\right)=\operatorname{Flux}\left(\left\{\widetilde{\phi}_{t}\right\}\right)$. Since $\operatorname{Flux}\left(\left\{\widetilde{\phi}_{t}\right\}\right)=$ 0 , there exists a function $\widetilde{F}: \widetilde{M} \rightarrow \mathbb{R}$ such that

$$
\int_{0}^{1} i_{\widetilde{X}_{t}} \widetilde{\omega} d t=d \widetilde{F}
$$

It is easy to verify that up to a constant $\left.\widetilde{F}\right|_{\partial M \times[1,+\infty)}$ may be chosen as:

$$
\widetilde{F}(m, z)=-\int_{0}^{1} z \alpha\left(\mathcal{X}_{t}\right)(m) d t=-z \int_{0}^{1} \alpha\left(\mathcal{X}_{t}\right)(m) d t
$$

Hence the Hamiltonian vector field $X_{\widetilde{F}}$ of $\widetilde{F}$ with respect to $\widetilde{\omega}$ is given by $\int_{0}^{1} \widetilde{X}_{t} d t$, and the restriction of it to $(\partial M \times[1,+\infty), d \Theta)$ is given by

$$
(m, z) \mapsto(\mathcal{X}(m, z), 0):=\left(\int_{0}^{1} \mathcal{X}_{t}(m) d t, 0\right) .
$$

This shows that the whole flow of $X_{\widetilde{F}}$ on $\widetilde{M}$, denoted by $\phi_{\widetilde{F}}^{s}$, exists and on $\partial M \times[1,+\infty)$ has the form: $\phi_{\widetilde{F}}^{s}(m, z)=\left(\chi^{s}(m), z\right)$, where $\chi^{s}$ is the flow of $\mathcal{X}$ on $\partial M$. The key point is

$$
\phi_{\widetilde{F}}^{s}(M)=M \quad \text { and } \quad \phi_{\widetilde{F}}^{s}(\partial M \times(1,+\infty))=\partial M \times(1,+\infty)
$$

for all $s \in \mathbb{R}$. Taking a strictly increasing smooth function $\eta:[0,1 / 4] \rightarrow[0,1]$ such that $\eta(0)=0, \eta(1 / 4)=1$ and $\eta^{\prime}(1 / 4)=0$, denoted by

$$
\widetilde{\psi}_{t}:= \begin{cases}\widetilde{\phi}_{\eta(t)} & \text { if } 0 \leq t \leq 1 / 4, \\ \widetilde{\phi}_{1} & \text { if } 1 / 4 \leq t \leq 3 / 4, \\ \phi_{\widetilde{F}}^{\eta(1-t)-1} \circ \widetilde{\phi}_{1} & \text { if } 3 / 4 \leq t \leq 1 .\end{cases}
$$

Setting $\widetilde{Z}_{t}:=\frac{d}{d t} \widetilde{\psi}_{t} \circ \widetilde{\psi}_{t}^{-1}$, it is a smooth family of vector fields on $\widetilde{M}$ and

$$
\int_{0}^{1} \widetilde{Z}_{t} d t=0
$$

From (118), (A.4), (A.5) it follows that

$$
\widetilde{\psi}_{t}(M)=M \quad \text { and } \quad \widetilde{\psi}_{t}((\partial M \times(1,+\infty))=\partial M \times(1,+\infty)
$$


for all $t \in[0,1]$. The straightforward computation shows that

(A.8) $\left.\widetilde{Z}_{t}\right|_{\partial M \times[1, \infty)}(m, z)= \begin{cases}\eta^{\prime}(t)\left(\mathcal{X}_{\eta(t)}(m), 0\right) & \text { if } 0 \leq t \leq 1 / 4, \\ 0 & \text { if } 1 / 4 \leq t \leq 3 / 4, \\ -\eta^{\prime}(1-t)\left(\int_{0}^{1} \mathcal{X}_{t}(m) d t, 0\right) & \text { if } 3 / 4 \leq t \leq 1 .\end{cases}$

Setting $\widetilde{Y}_{t}:=-\int_{0}^{t} \widetilde{Z}_{\lambda} d \lambda$, then

(A.9) $\left.\quad \widetilde{Y}_{t}\right|_{\partial M \times[1, \infty)}(m, z)= \begin{cases}\left(\int_{0}^{\eta(t)} \mathcal{X}_{s}(m) d s, 0\right) & \text { if } 0 \leq t \leq 1 / 4 \\ \left(\int_{0}^{1} \mathcal{X}_{s}(m) d s, 0\right) & \text { if } 1 / 4 \leq t \leq 3 / 4, \\ \left(\eta(1-t) \int_{0}^{1} \mathcal{X}_{s}(m) d s, 0\right) & \text { if } 3 / 4 \leq t \leq 1\end{cases}$

Let $\mathbb{R} \rightarrow \operatorname{Symp}_{0}(\widetilde{M}, \widetilde{\omega}), s \mapsto \widetilde{\theta}_{t}^{s}$ be the flow generated by $\widetilde{Y}_{t}$. Its existence is clear and is uniquely determined by

$$
\frac{d}{d s} \widetilde{\theta}_{t}^{s}=\widetilde{Y}_{t} \circ \widetilde{\theta}_{t}^{s}, \quad \widetilde{\theta}_{t}^{0}=i d .
$$

Moreover, since $\tilde{Y}_{0}=\tilde{Y}_{1}=0$ we get

$$
\widetilde{\theta}_{0}^{s}=\widetilde{\theta}_{1}^{s}=i d, \forall s \in \mathbb{R}
$$

The key point is that $\left.\widetilde{\theta}_{t}^{s}\right|_{\partial M \times[1,+\infty)}$ has the form

$$
\widetilde{\theta}_{t}^{s}(m, z)=\left(\hat{\theta}_{t}^{s}(m), z\right)
$$

for all $t \in[0,1], s \in \mathbb{R}$ and $(m, z) \in \partial M \times[1,+\infty)$. Here $\hat{\theta}_{t}^{s}: \partial M \rightarrow \partial M$. Setting $\widetilde{\varphi}_{t}:=\widetilde{\theta}_{t}^{1} \circ \widetilde{\psi}_{t}$ then it is easy to verify that $\operatorname{Flux}\left(\left\{\widetilde{\varphi}_{t}\right\}_{0 \leq t \leq T}\right)=0$ for every $T \in[0,1]$. Thus it is an Hamiltonian path starting from $i d$. Define another Hamiltonian path starting from $i d,[0,1] \rightarrow \operatorname{Ham}_{0}(\widetilde{M}, \widetilde{\omega}), t \mapsto \gamma_{t}$ by $\gamma_{t}=i d$ for $0 \leq t \leq 3 / 4$, and $\gamma_{t}=\phi_{\widetilde{F}}^{1-\eta(1-t)}$ for $3 / 4 \leq t \leq 1$. Then $t \mapsto \gamma_{t} \circ \widetilde{\varphi}_{t}$ is still an Hamiltonian path starting from $i d$. Moreover, when $s$ varies from 0 to 1 the path $\left(\gamma_{t} \circ \widetilde{\theta}_{t}^{s} \circ \widetilde{\psi}_{t}\right)_{t \in[0,1]}$ starting from $i d$ varies from $\left(\gamma_{t} \circ \widetilde{\psi}_{t}\right)_{t \in[0,1]}$ to $\left(\gamma \circ \widetilde{\varphi}_{t}\right)_{t \in[0,1]}$ with fixed endpoints. Since $\gamma_{t}(m, z)=(m, z)$ for $0 \leq t \leq 3 / 4$, and $\gamma_{t}(m, z)=\left(\chi^{1-\eta(1-t)}(m), z\right)$ for $3 / 4 \leq t \leq 1$, it is easily 
checked that

(A.10) $\left.\quad \gamma_{t} \circ \widetilde{\theta}_{t}^{s} \circ \widetilde{\psi}_{t}\right|_{\partial M \times[1,+\infty)}(m, z)$

$$
= \begin{cases}\left(\hat{\theta}_{t}^{s}\left(\Phi_{\eta(t)}(m), z\right)\right. & \text { if } 0 \leq t \leq 1 / 4, \\ \left(\hat{\theta}_{t}^{s}\left(\Phi_{1}(m)\right), z\right) & \text { if } 1 / 4 \leq t \leq 3 / 4 \\ \left(\chi^{1-\eta(1-t)} \circ \hat{\theta}_{t}^{s} \circ \chi^{\eta(1-t)-1} \circ \Phi_{1}(m), z\right) & \text { if } 3 / 4 \leq t \leq 1 .\end{cases}
$$

From these it follows that when $s$ varies from 0 to 1 the path $\left(\gamma_{t} \circ\right.$ $\left.\left.\widetilde{\theta}_{t}^{s} \circ \widetilde{\psi}_{t}\right|_{M}\right)_{t \in[0,1]}$ varies from $\left(\left.\gamma_{t} \circ \widetilde{\psi}_{t}\right|_{M}\right)_{t \in[0,1]}$ to the Hamiltonian path $\left(\left.\gamma_{t} \circ \widetilde{\varphi}_{t}\right|_{M}\right)_{t \in[0,1]}$ with fixed endpoints. But $\left.\gamma_{t} \circ \widetilde{\psi}_{t}\right|_{M}=\phi_{\eta(t)}$ for $0 \leq t \leq 1 / 4$, and $\left.\gamma_{t} \circ \widetilde{\psi}_{t}\right|_{M}=\phi_{1}$ for $1 / 4 \leq t \leq 1$. That is, $\left.t \mapsto \gamma_{t} \circ \widetilde{\psi}_{t}\right|_{M}$ is only an reparametrization of the path $t \mapsto \phi_{t}$. This completes the proof of Theorem A.

\section{References.}

[ALP] M. Audin, F. Lalonde and L. Polterovich, Symplectic rigidity: Lagrangian Submanifolds, in 'Holomorphic Curves in Symplectic Geometry,' Birkhäuser Verlag, Progress in Math., 117 (1994), 271318.

[F] A. Floer, The unregularized gradient flow of the symplectic action, Comm. Math. Pure Appl. Math., 41 (1998), 775-813.

[FO] K. Fukaya and K. Ono, Arnold conjecture and Gromov-Witten invariant, Topology, 38 (1999), 933-1048.

[Gi] B. Givental, Eqivariant Gromov-Witten Invariants, preprint, 1996.

[Gr] M. Gromov, Pseudoholomorphic curves in symplectic manifolds, Inv. Math., 82 (1985), 307-347.

[Hu] D. Husemoller, Fibre Bundles, McGraw-Hill Series in Higher Mathematics., 1966.

[K1] M. Kontsevich, Enumeration of rational curves via torus actions, in 'Moduli space of surface', H. Dijkgraaf, C. Faber and v.d.G. Geer, Editors, 1995, Birkhäuser, Boston, 335-368. 
[K2] M. Kontsevich, Homological Algebra of Mirror Symmetry, Proceedings of the International Congress of Mathematicians, Zürich, Switzerland, 1994.

[KM] M. Kontsevich and Y. Manin, GW classes, Quantum cohomology and enumerative geometry, Comm.Math.Phys., 164 (1994), 525562 .

[L] P. Lu, A rigorous definition of fiberwise quantum cohomology and equivariant quantum cohomology, Comm.Anal. Geom., 6 (1998), 511-588.

[Le] Hong-Van Le, Topology of symplectomorphism groups and pseudoholomorphic curves, preprint, MPI, 1997.

[LeO] Hong-Van Le and K. Ono, Cup-length estimates for symplectic fixed points. Contact and symplectic geometry, (Cambridge, 1994), 268295, Publ. Newton Inst., 8, Cambridge Univ. Press, Cambridge, 1996.

[LMP] F. Lalonde, D. McDuff and L. Polterovich, Topological rigidity of Hamiltonian loops and quantum homology, Inv. Math., 135 (1999), 369-385.

[LT1] J. Li and G. Tian, Virtual moduli cycles and Gromov-Witten invariants of algebraic varieties, J. Amer. Math. Soc., 11 (1998), 119-174.

[LT2] J. Li and G. Tian, Virtual moduli cycles and Gromov-Witten invariants of general symplectic manifolds, Topic in symplectic 4manifolds (Irvine, CA, 1996), 47-83, First Int. Press Lect. Ser., I, Internatinal Press, Cambridge, MA, 1998.

[Lu1] G.C. Lu, The Arnold Conjecture for a Product of Weakly Monotone Manifolds, Chinese. J. Math., 24(2) (1996), 145-158.

[Lu2] G.C. Lu, Weinstein Conjecture on Some Symplectic Manifolds Containing the Holomorphic Spheres, Kyushu Journal of Mathematics., 52(2) (1998), 331-351.

[Mc1] D. McDuff, Examples of symplectic structures, Inv. Math., 89 (1992), 13-36. 
[Mc2] D. McDuff, Quantum homology of fibrations over $S^{2}$, preprint SG 9905092.

[McSa1] D. McDuff and D. Salamon, J-holomorphic curves and quantum cohomology, University Lec. Series, 6, AMS.

[McSa2] D. McDuff and D. Salamon, Introduction to Symplectic Topology, Oxford University Press, 1995.

[Mo] D.R. Morrison, Mathematical Aspects of Mirror Symmetry, preprint.

[PW] T. Parker and J. Wolfson, Pseudo-holomorphic maps and bubble trees, J. Geom. Analysis., 3 (1993), 63-98.

[R1] Y. Ruan, Topological Sigma model and Donaldson type in Gromov theory, Math. Duke. Jour., 83(2) (1996), 461-500.

[R2] Y. Ruan, Symplectic topology on algebraic 3-folds, J. Diff. Geom., 39 (1994), 215-227.

[R3] Y. Ruan, Virtual neighborhood and pseudo-holomorphic curves, preprint.

[RT1] Y. Ruan and G. Tian, A mathematical theory of quantum cohomology, J. Diff. Geom., 43(2) (1995).

[RT2] Y. Ruan and G. Tian, Higher genus symplectic invariants and sigma model coupled with gravity, Inv. Math..

[Se1] P. Seidel, $\pi_{1}$ of symplectic automorphism groups and invertibles in quantum homology rings, Geom. Funct. Anal., 17(6) (1997).

[Se2] P. Seidel, Symplectic automorphisms of $T^{*} S^{2}$, preprint DG 9803084.

[Sie] B. Siebert, Gromov-Witten invariants for general symplectic manifolds, preprint, 1996.

[Sik] J.C. Sikorav, Some properties of holomorphic curves in almost complex manifolds, in 'Holomorphic Curves in Symplectic Geometry', Birkhäuser Verlag, Progress in Math., 117 (1994), 165-189.

[W1] E. Witten, Topological sigma models, Comm. Math. Phys., 118 (1988). 
[W2] E. Witten, Two dimensional gravity and intersection theory on moduli space, Surveys in Diff. Geom., 1 (1991), 243-310.

[Ye] R. Ye, Gromov's compactness theorem for pseudo-holomorphic curves, Trans. Amer. Math. Soc., 343 (1994), 671-694.

Department of Mathematics

BEIJING NORMAL UNIVERSITY

BeiJing 100875, P. R. China

E-mail address: gclu@bnu.edu.cn

ReCeived June 16, 1999. 